EUROPEAN ORGANIZATION FOR NUCLEAR RESEARCH

CERN-EP/2002-038

22 February 2002

\title{
OBSERVATIONAL EVIDENCE FOR GRAVITATIONALLY TRAPPED MASSIVE AXION(-LIKE) PARTICLES
}

\author{
L. DiLella ${ }^{1, *)}$ and K. Zioutas ${ }^{1,2)}$
}

\begin{abstract}
Several unexpected astrophysical observations can be explained by gravitationally captured massive axions or axion-like particles, which are produced inside the Sun or other stars and are accumulated over cosmic times. Their radiative decay in solar outer space would give rise to a 'self-irradiation' of the whole star, providing the time-independent component of the corona heating source (we do not address here the flaring Sun). In analogy with the Sun-irradiated Earth atmosphere, the temperature and density gradient in the corona-chromosphere transition region is suggestive for an omnipresent irradiation of the Sun, which is the strongest evidence for the generic axion-like scenario. The same mechanism is compatible with phenomena like the solar wind, the X-rays from the dark-side of the Moon, the X-Ray Background Radiation, the diffuse X-ray excesses (below $\sim 1 \mathrm{keV}$ ), the non-cooling of oldest Stars, etc. A temperature of $\sim 10^{6} \mathrm{~K}$ is observed in various places, while the radiative decay of a population of such elusive particles mimics a hot gas, which fits unexpected astrophysical X-ray observations. Furthermore, the recently reconstructed quiet solar X-ray spectrum during solar minimum supports this work, since it covers the expected energy range, and it is consistent with the result of a simulation based on Kaluza-Klein axions above $\sim 1 \mathrm{keV}$. The derived axion luminosity $\left(L_{a} \approx 0.16 L_{\odot}\right)$ fits the cosmic energy density spectrum and is compatible within $2 \sigma$ with the recent SNO result, showing the important interplay between any exotic energy loss mechanism and neutrino production. At lower energies, using also a ROSAT observation, only $\sim 3 \%$ of the X-ray intensity is explained. Data from orbiting X-ray Telescopes provide upper limits for particle decay rates 1 AU from the Sun, and suggest new types of searches on Earth or in space. In particular, X-ray observatories, with an unrivalled equivalent fiducial volume of $\sim 10^{3} \mathrm{~m}^{3}$ for the 0.1 - $10 \mathrm{keV}$ range, can search for the radiative decay of new particles even from existing data. This work introduces the elongation angle of the X-ray Telescope relative to the Sun as a relevant new parameter.
\end{abstract}

\section{(To appear in Astroparticle Physics)}

1) CERN, CH-1211 Geneva 23, Switzerland.

2) Physics Department, University of Thessaloniki, GR 54124 Thessaloniki, Greece.

*) E-mails: Luigi.Di.Lella@cern.ch, Konstantin.Zioutas@cern.ch 


\section{INTRODUCTION}

The direct detection of dark-matter (DM) particles has proved elusive since the first gravitational observation of non-luminous matter in the Universe. So far, the outcome of the intense experimental and theoretical work in the field of dark matter during the last $\sim 20$ years was the birth of the new discipline of astroparticle physics.

In this work, a rather old question is reconsidered as to whether a large number of as yet unexplained astrophysical phenomena occur because of the involvement of novel very weakly interacting particles or additional as yet unknown properties of existing particles. For example, in order to explain the ionization state of the intergalactic medium, and the anomalous ionization of the interstellar medium [1], speculations included a widespread source of ionizing UV-photons from the electromagnetic decay of real or hypothetical exotic particles (clustered in haloes) [2], e.g., massive dark-matter neutrinos [3, 4]; however, observations appear to have ruled out this model [1, 5]. Considerations based on axions apply as well, invoking either its $2 \gamma$ decay mode or its coherent conversion to a photon inside astrophysical electric/magnetic fields via the Primakoff effect $[$.

Recently, in order to explain the as yet unknown underlying mechanism(s) of the Gamma Ray Bursts, massive axions with properties far beyond the widely accepted theoretical axion concepts have been considered, providing a built-in dissipationless energy transfer mechanism from the hypothetical energy generating core to the outside layers some 100-1000 km away [9]. Even though none of such intriguing ideas has been established so far [10], an additional electromagnetic energy source in the cosmos seems to be necessary. Structure formation in warm dark matter (WDM) cosmological models [1] provides a lower limit to the mass of the WDM particle candidate of $\sim 0.75 \mathrm{keV}$, but with a radiative decay lifetime as large as $\sim 10^{16} \times$ Hubble time; however, halos in Galaxies and clusters of Galaxies can be an enormous "fiducial volume" of DM particles.

In this work, we argue that the photon emission of some hypothetical particles, referred to more generally as 'axion-like', could be involved in different unexplained astrophysical observations. An extensive search of astrophysical literature has been undertaken, which includes also some 10-50 years old observations. In this work we focus mainly on :

a) the solar corona problem and related observations;

b) the observed X-rays from the direction of the dark side of the Moon;

c) the soft-X-ray background radiation;

d) the (diffuse) soft X-ray excess phenomenon;

e) first simulation results in the frame of an axion scenario.

Following the reasoning of this work, we also suggest performing a specific axion search in a new type of experiment, either on the surface (and in space) or underground, aiming directly at detecting the $2 \gamma$ decay / interaction mode. The alternative case of a single photon emission seems to be beyond the present sensitivity of an Earth-bound detector and only orbiting X-ray telescopes could be considered. In addition, some astrophysical

1) a) The absence of a monochromatic axion line from the night Sky expected from the $a \rightarrow \gamma \gamma$ decay of relic axions in the visible [6] almost excluded an axion rest mass in the $\sim 1-10 \mathrm{eV}$ range. b) Until recently, a conventionally expected thermal X-ray spectrum from solar axions converted inside the solar magnetic fields with mean energy of $\sim 4.4 \mathrm{keV}$ could not have been disentangled from the derived solar X-ray spectrum (e.g. Ref. 7). In any case, such a mechanism could not explain the main observations addressed in this work, because such converted axions give rise to photons always emitted away from the Sun. Moreover, even in the scenario of massive axions from the Sun, the possibility to detect photons emitted in flight through axion decays was considered to be "presumably indistinguishable from the general background radiation" [8]. 
measurements could be reconsidered or re-analysed. X-ray space detectors could operate also as sensitive orbiting axion telescopes.

\section{OBSERVATIONAL EVIDENCE}

In this section we present some not yet understood astrophysical phenomena or experimental results, which can be explained within the frame of the same axion-like scenario.

\subsection{Solar corona}

The existence of the solar corona has been known for more than 100 years. However, solar X-rays have been measured only over the last 50 years, providing an unexpected and anomalously high temperature [12, 13, 14]. The corona is the only atmospheric layer of the Sun that emits almost thermally in X-rays [15]. The average temperature of the quiet-Sun corona is $\sim 2 \cdot 10^{6} \mathrm{~K}$. Although its phenomenology is well understood, the coronal heating remains one of the most puzzling problems in solar physics 112, 14, 16, 17, 18, 19, 20, 21, 22, 23, 24]. Recently, reconstructed X-ray energy spectra have been published, providing additional valuable information about our Sun, such as temperature, solar cycle dynamics, etc. [16]. The quiet-Sun X-ray luminosity represents only a fraction of $\leq 10^{-7}$ of the total solar luminosity $\left(L_{\odot}^{x} \approx 2 \cdot 10^{27} \mathrm{erg} / \mathrm{s}\right.$ at solar maximum [25, 26, 27], which never drops to zero). Therefore, energy balance problems are irrelevant 2 . Thus, the main puzzles with the solar corona and the very thin [30] transition region (TR) between the corona and the underlying chromosphere (the least understood region of the solar atmosphere [31]) are the following :

a) The hot corona cannot be in equilibrium with the $\sim 300$ times cooler solar surface underneath (violating thus the second law of thermodynamics [32]), which emits an almost perfect blackbody radiation in the visible [33] (Fig. 1b).

b) In order to maintain the quiet Sun high temperature corona, some nonthermally supplied energy must be dissipated in the upper atmosphere [34], which is lost as a large inward heat flux into the transition region, solar wind energy and transition region pressure [35, 36]; these processes are thermodynamically allowed, because of the purported coronal temperature increase with height.

c) One must explain the abrupt (in some models [37] even within $\sim 100 \mathrm{~km}$ ) temperature increase (from $\sim 8 \cdot 10^{3} \mathrm{~K}$ to $\sim 5 \cdot 10^{5} \mathrm{~K}$ ) in the chromosphere/corona transition region (Fig. 1a), against physical expectation [38, 39, 40, 41] 3).

This is 'the solar corona problem', which is well stated in a recent review article [29] : "everything above the photosphere ..... would not be there at all".

2) In this work we refer to the quiet Sun, where also nano-flares occur [28] and they could account for a significant fraction of the coronal heating [29]. The X-ray power of the active Sun is higher by a factor of $\sim 20$, while its temperature of $\approx 8-20 \mathrm{MK}[16,27$ is equal to that of the Sun core.

3) Note that most of the solar light comes from the $\sim 100 \mathrm{~km}$ visible photosphere $(\sim 5800 \mathrm{~K})$. Above that, there is the chromosphere which is astonishingly hot (up to $25000 \mathrm{~K}$ ), and above that, the corona (up to a few MK and locally much more [39]). Corona X-rays have been observed out to $\sim 1$ solar radius. The outer corona expands into the interplanetary space and slowly cools off. At $\sim 1-100 \mathrm{AU}$ the temperature is still $\sim 10^{5} \mathrm{~K}[42]$ (see also R.F. Stein in Ref. [38]). If the corona is heated by thermal processes, it could not be hotter than the photosphere. The second law of thermodynamics precludes thermal transfer from the relatively cool photosphere to the much hotter corona 32]. Therefore, it must be heated by some non-thermal process [38, 42], e.g., by electromagnetic energy input [32]. Obviously, only below a certain density can the energy input (whatever the required nonthermal energy transfer mechanism is in reality) be sufficient to increase the temperature or trigger an unexpected process. 
The step-like change of the corona temperature coincides in space with a similar (opposite) density gradient (Fig. 1a), thus suggesting a common origin. Qualitatively, this peculiar behaviour of the Sun atmosphere is suggestive for some external irradiation (pressure) acting on the whole Sun, and only such a configuration can cause the 'compression' and the heating of the intervening solar atmosphere (= corona region). Depending on the energy, these photons are absorbed mainly at a certain depth (as seen from outside the Sun) due to the exponential increase of the density with decreasing height of the solar atmosphere (see Fig. 1a and Ref. [38]). One should keep in mind that the density at the place where both steps occur is $\sim 10^{-(12 \pm 1)} \mathrm{g} / \mathrm{cm}^{3}$, i.e. an excellent vacuum, which actually does not facilitate a conventional explanation of this observation.

The column density in the solar atmosphere at an altitude of $\sim 2000 \mathrm{~km}$ down to $\sim 1000 \mathrm{~km}$ is $\sim 10 \mu \mathrm{g} / \mathrm{cm}^{2}$ to $\sim 1 \mathrm{mg} / \mathrm{cm}^{2}$, respectively 43. We take the most abundant 11 solar elements in neutral form ( $\mathrm{H}, \mathrm{He}, \mathrm{C}, \mathrm{N}, \mathrm{O}, \mathrm{Ne}, \mathrm{Mg}, \mathrm{Si}, \mathrm{Ar}, \mathrm{Ca}$, and Fe), in order to make a rough estimate of the energy of incident photons which can be absorbed at such depths of the solar atmosphere. Photons with an energy below $\sim 50$ to $350 \mathrm{eV}$, hitting the solar surface perpendicularly (i.e. at the minimum absorption thickness) can be absorbed at the considered depths [46]. The higher energetic X-rays penetrate deeper into the underlying chromosphere, where the onset of the anomalous temperature rise above the photosphere occurs. Remarkably, the observationally reconstructed solar X-rays [16] above $\sim 1 \mathrm{keV}$ have a mean free path into the Sun atmosphere of $\sim 10 \mathrm{mg} / \mathrm{cm}^{2}\left(E_{\gamma} \approx 1\right.$ $\mathrm{keV})$ and $\sim 1 \mathrm{~g} / \mathrm{cm}^{2}\left(E_{\gamma} \approx 6\right.$ to $\left.8 \mathrm{keV}\right)$, corresponding to heights of $\sim 700 \mathrm{~km}$ and $\sim 200$ $\mathrm{km}$, respectively. Interestingly, at a height of $\sim 500 \mathrm{~km}$ there is a temperature minimum of $\sim 4000^{\circ} \mathrm{K}$ [31], while $\mathrm{X}$-rays below a few keV barely reach this place; a wide energy distribution of incident X-rays above $1-2 \mathrm{keV}$ [16] could be related to the relatively slow increase of the chromosphere's temperature with height. Note that the coronal heating problem cannot be solved without including energization processes in the chromosphere and the transition region 47 .

All these findings and problems associated with the solar atmosphere can be reconciled by assuming axion(-like) particles which stream out of the interior of the Sun and undergo photon decay, giving rise to the occurence of otherwise unexpected nonthermal phenomena. The photons from the $a \rightarrow \gamma \gamma$ decay are emitted isotropically, i.e. also towards the Sun, resulting to an external illumination of the whole solar atmosphere the dense interior of the Sun the axions can only have a negligible impact due to energy considerations, thus avoiding any conflict with the generally accepted solar model(s). However, above some altitude, i.e. below a certain density, the thermodynamical equilibrium starts getting disturbed because of an additional external energy input, coming -ironically - from the Sun itself. Without taking into account this energy source, the temperature appears to increase to thermodynamically not allowed values across the chromosphere and in particular across the narrow transition region.

The striking similarity of the temperature and density dependence on the altitude between the solar irradiation of the Earth atmosphere and Sun atmosphere [33], in par-

4) Observations suggest that there is considerable roughness and variability of the transition region 29, 44], which might consist of many sharp temperature jumps in the different places, but not always at the same height 45] (see also ref. [37). Therefore, we use first these two penetration depths in our order of magnitude numerical calculation of the photoelectric absorption of the soft X-rays, in order to derive approximately the X-ray energy from the assumed axion decays around the Sun.

5) The bombardement of the Sun by cosmic material has been proposed some 50 years ago, in order to explain the Sun's dynamical behaviour, but this scenario was later ruled out [48. 
ticular around the transition region, strongly supports the conclusion of an external illumination of the Sun in the UV-X-ray region (compare Fig. 1a with Fig. 1d). The observation made in the atmosphere of Venus [49] seems to be relevant too : due to the solar irradiation, its nightside density $\left(\rho \approx 10^{-15} \mathrm{~g} / \mathrm{cm}^{3}\right.$ at a height of $\left.170-190 \mathrm{~km}\right)$ and temperature increase during local day time by a factor of 10 and $30 \%$, respectively. The photoionization rate peaks at an altitude of $\sim 140 \mathrm{~km}$ [50]. The planetary absorption depth of the solar radiation reflects the energy of this radiation 33 (see Fig. 1c). Similarly, we conclude that for the case of the solar atmosphere the photons emitted towards the Sun from the decay of nearby exotic particles $(a \rightarrow \gamma \gamma)$ must have an energy mainly around $\sim 200 \mathrm{eV}$ and also of a few $\mathrm{keV}$. This fits the appearance of the abrupt TR and the rather slowly increasing chromosphere temperature with height [51, 52]. Moreover, Monte Carlo simulations of X-rays irradiated stellar atmospheres [53] and accretion disks [54] predict a similar stepwise temperature gradient as those shown in Fig. 1 for the Earth and Sun atmospheres.

In addition, the following observation is compatible with the scenario of an external whole Sun X-ray irradiation. In coronal holes, where the electron density is by a factor of 2 to 3 lower than normal quiet-Sun regions [52, 55, 56], it was found that the thickness of the transition region is also larger than in near quiet-Sun regions [57] (see also ref. [58] p. 154).

\section{Related observations.}

1) The solar wind : the suggested axion scenario could also explain the apparently not so unrelated origin of the solar wind, whose acceleration mechanism is one of the outstanding problems in solar physics [59]. The proton wind has a low velocity component $(\sim 350-$ $500 \mathrm{~km} / \mathrm{s}$ ) and a fast one (above $\sim 750 \mathrm{~km} / \mathrm{s}$ ) [38, 60, 61]. The high speed solar wind outflows are accelerated very close to the solar surface [62]. The solar wind originates from the coronal holes, which are open magnetic field regions with a temperature of $\sim 1 \mathrm{MK}$ [63] (see also ref. [38]).

We use in what follows the low X-ray energy derived above $\left(E_{\gamma} \sim 200 \mathrm{eV}\right)$ in our attempt to reproduce main corona features, while the hard X-rays apply as well; the ejected photoelectrons basically carry away the energy of the photon, i.e. their velocity is $v_{e} \leq 8500 \mathrm{~km} / \mathrm{s}$. Let the number of the photoelectrons be equal to that of the protons. Then, the onset of the equilibration [64] between electrons and protons results to a proton kinetic energy of $T_{p} \leq 200 \mathrm{eV}$, i.e. a proton velocity $v_{p} \sim 200 \mathrm{~km} / \mathrm{s}$. This certainly not rigorous estimate provides a rather reasonable value compared to the measured proton peak at $v_{p} \approx 500 \mathrm{~km} / \mathrm{s}$ [61. Note that the derived electron velocities leave room for much higher ion velocities, including the high speed proton wind (up to $\sim 1200 \mathrm{~km} / \mathrm{s}$ ), which makes only $\sim 10^{-5}-10^{-6}$ of the bulk proton intensity. At this level, some protons might acquire more speed from the ambient electron gas, e.g., due to a locally smaller proton-to-electron density ratio [0], or some other transient conditions.

2) The stellar coronae: like our Sun, many other Stars also have outer layers that are hotter than the underlying photosphere, giving rise to corona formation. Observations suggest that the corona of the Sun and the stellar coronae have common heating

6) Ions merged with a faster moving electron bunch exchange momentum-energy. If the number of the ions is relatively small, they may reach the velocity of the surrounding electron cloud, somehow similar to the old collective ion accelerator concept 65. Also, the electron cooling in accelerators is based on the "heat exchange" between the ions and the electron gas [66. 
mechanism(s) [12, 42, 67]. Therefore, the solar corona problem is not just a solar problem [12, 42, 67] and our conclusions based on solar observations become rather global. Measurements of UV and X-rays have revealed the ubiquitous presence of solar-like transition regions and coronae 68] in outer atmospheres of completely different types of Stars -from the coolest M-type dwarfs (mass $\left.\leq 0.3 M_{\odot}\right)$ ) to evolved giants- being "perhaps disappointing similar" [5]. Thus, cool Stars without a surrounding corona either do not exist or are very rare 25]. Therefore, the question about what heats the coronae of the Sun or other cool stars is one of the outstanding problems in astrophysics [68, 70, 77].

3) The old Stars: it was pointed out already in 1926 by Eddington that a Star in a steady state (= in radiative equilibrium) necessarily re-emits completely the radiation falling on it [72. The incident radiation maintains the surface layers at a temperature higher than they would otherwise have [73], and they radiate back in space the absorbed energy in addition to that which they would radiate in the absence of the incident radiation. As with the suggested axion-related irradiation of the Sun, a similar self-irradiation of the Star by unstable and long-lived axion-like particles can also take place. Then, some related phenomena should have been observed - even unintentionally - also from the oldest astrophysical objects whose activity in general decreases with age [74]. Thus, we looked for observational evidence from the following fossil Stars :

a) Millisecond Pulsars with a spin-down age of $\sim 1$ to $10 \mathrm{Gyr}$; according to the Standard Models for cooling, without a strong reheating mechanism [75], they are too old to expect any detectable thermal X-ray emission $[76$. In one case, with the observed EUV emission, all conventional possibilities were ruled out [75] 8). X-ray emission of as yet unknown origin has also been detected from a group of msec Pulsars 77.

b) White Dwarfs (WDs) with an age above $\sim 10 \mathrm{Gyr}$, which are the very last evolution phase of a Star (as our $\sim 4.6$ Gyr old Sun will be in $\sim 6$ Gyr). We give here a few findings for these Earth-size objects with mass $\mathrm{M} \approx 0.6 M_{\odot}$ :

1. Coronal X-rays have been detected from a WD with a temperature of $(0.2-0.3) \cdot 10^{6} \mathrm{~K}$ (78) ए).

2. The abrupt and steep decrease of the number of WDs at very cool temperatures (below $\sim 4000 \mathrm{~K}$ ) cannot easily be explained as a cooling effect, i.e. WDs have had not sufficient time to cool [80]. A recent proposal [81] to resolve the mystery shows a lack of understanding of the actual physical conditions.

3. Unexpectedly, WDs appear to be usually much younger than Pulsars in old binary systems, which should have the same age; in one case the cooling models provided 0.3 Gyr for the WD, while the spin-down age of the Pulsar was $\sim 7$ Gyr. This is suggestive for an additional heating source, which slows down the cooling of the WDs [82].

Qualitatively, such diverse observations are in favour of axion-like particles gravitationally trapped by the Star itself. If these particles are sufficiently long lived, a part of them can

7) Interestingly, according to ref. [69, such Stars being fully convective could not sustain a dynamo which was thought to be necessary for solar activities. And, these two experts add : Thumbing their nose at theory, these Stars still possess energetic coronas".

8) Magnetic monopole catalyzed nucleon decay was considered as the additionally required heating source.

9) If the captured population of particles around a Star is of Kaluza-Klein axion-type [79], then the older the object the less massive axions survive, implying thus a decreasing coronal temperature with time. 
survive the evolutionary steps of the Star, giving rise to astrophysical phenomena like winds, stellar coronae, X-ray/EUV emission, non-cooling of old Stars, etc. The X-rays from the decay of gravitationally captured axions can mimic thermal emission and could lead to a wrong interpretation of the black body emission from the Star surface. For example, the recently claimed evidence for a compact (quark) star [83] could be alternatively explained by the mechanism suggested in this work.

\subsection{X-rays from the direction of the dark side of the Moon}

We reconsider here one lunar experiment performed by the ROSAT [84] orbiting Xray telescope in 1990, which provided the first X-ray 'photo' (Fig. 2) from the sunlit side of the Moon [85. In fact, with the X-ray telescope field-of-view aligned towards the Moon, this configuration eliminated the $\sim 3.3 \times$ brighter diffuse $\mathrm{X}$-ray background radiation (XRB) (see Fig. 4 in Ref. 855). ROSAT has also observed rather intense X-ray emission coming from the optically dark side of the Moon (Fig. 2) : its shadow emits X-rays at a level as high as 1\% and 30\% compared to that of the bright side of the Moon and of the XRB radiation, respectively. It is interesting to note that all these three components are extracted from the same X-ray image, and they have a quite similar spectral shape (Fig. 3).

Within the axion scenario (see section 2.5), X-rays should come (obliquely) from the Sun neighbourhood. An (isotropic) X-ray emission will mimic scattering of solar Xrays by gas, plasma, dust, etc. [58], which is a well-known phenomenon in astrophysics. The low-energy spectrum of the ubiquitous diffuse X-ray background radiation (XRB) overlaps with the solar one (see Fig. 3), while its intensity is by no means negligible. Thus, reprocessing of solar X-rays by matter in outer solar space and the XRB must be taken into account, in order to correctly interpret existing data or to design a new measurement following the reasoning of this work.

The interaction of the solar wind with the dark surface of the Moon has been suggested as the source of these lunar X-rays [85]. Such an explanation should actually be the natural one, but the estimated absolute intensity seemed not to be completely satisfactory. Later, EUV lunar data [86] have shown that the decline in albedo from FUV to X-ray wavelengths was more shallow than expected, and this was considered as a possible evidence of lunar X-ray fluorescence (not reflection). Furthermore, Ref. 87] reaches the tentative conclusion that there was no emission from the Moon itself, without excluding the opposite [85. In addition, a preliminary re-analysis [88] of this experimental result reached the following tentative conclusions $\square$ :

a) The dark side of the Moon is brighter than expected by a factor of more than 10 .

b) This excess is consistent with the effect of an X-ray emitting region around the Earth.

From all these conclusions, it seems that a conventional reasoning has difficulties to convisingly explain this (unexpected) lunar X-ray shadow. However, these X-ray photons from the direction of the dark side of the Moon (Fig. 3B) fit the solar axion scenario (with the axion energy being obviously twice that of Fig. 3B). It is interesting to note that the energy of the bulk of these single photons (Fig. 3B) from the dark Moon direction $(\sim 100$ to $\sim 400 \mathrm{eV})$ coincides with that discussed in Section $2.1(\sim 50$ to $\sim 350 \mathrm{eV})$, while the observed X-ray spectrum extends up to $\sim 2 \mathrm{keV}$; beyond this energy the response curve of ROSAT diminishes.

10) The purpose was actually to propose a possible extra component of the soft XRB [88]. 
In any case, from this lunar observation we derive the best experimental upper limit for the radiative decay rate of exotic particle candidates at the site of the Earth. This is a useful input for (future) experimental searches of this kind (see Section 3).

\subsection{The X-ray background radiation (XRB)}

The origin of the diffuse XRB radiation accidentally discovered in 1962 [89, 90] remains a mystery [91] and is the most enduring problem in X-ray astrophysics [92, 93]. From a wealth of data about its galactic component below $1 \mathrm{keV}$, the existence of a $\sim 100$ pc extended optically thin local $\sim 10^{6} \mathrm{~K}(\sim 0.1 \mathrm{keV})$ hot plasma has been invented, which fills the 'Local Hot Bubble' (LHB) [94] : a soft X-ray-emitting region around the Sun, essentially free of neutral gas [95, whose origin is unknown [94, 96, 97]. The existence of a hot gas as a major constituent of the interstellar medium follows from the observed UV resonance absorption lines from highly ionized species such as O-VI [98], while recent work 97 suggests either a much smaller temperature of the gas, or that it is hot but also extremely metal deficient. Unexpectedly, no emission lines from such a hot plasma were detected, and this result is disturbing [5]. However, in the suggested axion scenario, a radiatively decaying population of trapped axions mimics a hot plasma (component), and it remains hidden.

The soft XRB temperature component of $\sim 10^{6} \mathrm{~K} 99$ is encountered also in various other places in the Universe. In the $\sim 0.1-0.8 \mathrm{keV}$ band the XRB intensity exceeds the extrapolated higher energy part [93, 100, 101, 102, 103, 104]; a broad diffuse soft excess (below $\sim 1 \mathrm{keV}$ ) seems to be established [105]. Orbiting X-ray telescopes have found ubiquitous plasmas above $\sim 10^{6} \mathrm{~K}$, whose heating, however, is poorly understood [106], while most of the baryons in the Universe are thought to reside in intergalactic space at temperatures $\sim 10^{6 \pm 1} \mathrm{~K}[107]$.

Observations show that the XRB is comprised of the integrated contributions from a large number of discrete sources (including Clusters of Galaxies) [108, 109, 110], with only a small portion remaining unexplained [111]. In addition, the recently obtained XRB spectrum [100, 102, 111], with a bump at $\sim 0.7 \mathrm{keV}$, coincides in energy with the bulk of the spectrum of solar-type Stars [7]. The (same) axion scenario should apply though not only to our Sun. In summary, the following observations are in favour of an axion related scenario as the origin of the (soft) XRB :

a) The quiet-Sun corona [16, 34, 39, 112] and the coronae of other Stars [106] (most late-type Stars are surrounded by hot (above $10^{6} \mathrm{~K}$ ) corona [74).

b) The Local Hot Bubble 113, 114, 115, and the galactic diffuse X-ray emission beyond it [108, 114, 115, 116].

c) Intergalactic matter, which requires a significant amount of non-gravitational heating (see below).

d) A number of X-ray Clusters of Galaxies are better modelled using a second cooler component of $\sim 10^{6} \mathrm{~K}$ [117]. Also, a soft excess $(\sim 0.1-0.4 \mathrm{keV})$ from the central Galaxy M87 of the Virgo cluster has been established; in order to prevent the gas from rapidly cooling, some as-yet-undiscovered heat source must be at work 118, 119, 120, 121.

For the extragalactic component of the XRB above $\sim 1 \mathrm{keV} \mathrm{104,} \mathrm{where} \mathrm{the} \mathrm{bulk}$ of its energy density resides [122], a widespread optically thin and very hot uniform intergalactic medium (temperature $\sim 10-40 \mathrm{keV}$ ) has been ruled out by the lack of distortion of the Planck spectrum of the $2.7 \mathrm{~K}$ Cosmic Microwave Background radiation (CMB) 
109, 123, 124, 125] : the Compton scattering with the very hot plasma electrons should generate a change in the CMB temperature (Sunyaev-Zel'dovich effect) [104. For this $\mathrm{XRB}$ radiation, an axion scenario could also be at work, requiring however accordingly hot places in the Sky.

\subsection{The soft X-ray excess phenomenon}

Decay photons from axions gravitationally captured in the potential wells of galaxies, or clusters of galaxies have been searched for in the past as narrow lines in microwaves [126 as well as in the visible [127]. By contrast, the radiative decay of massive axions or axion-like particles should result primarily in a diffuse photon emission with a broad energy distribution reflecting the temperature of their place of birth (e.g. the Sun core, or the accreting disk of a massive black hole, etc.). In addition, if the observed X-rays happen to be in excess of the conventionally expected ones, we are led to further consider whether such an observation is in favour of this work 1 ). As usually in astrophysics, there is, however, no luck of alternative explanations. Therefore, we address (throughout this work) the mostly striking findings we are aware of, which lack any clear explanation.

We emphasize, among many astrophysical observations of a soft X-ray excess [129, 130], the recent discovery by ROSAT of the so far brightest diffuse soft X-ray excess from a cluster of Galaxies [131] [2)] : since all the competing models are confronted with serious problems, a major effect must be at work in the intergalactic medium that has hitherto been completely ignored. In addition, models of cluster formation, in which the intergalactic gas falls into the dark matter dominated gravitational well, fail to reproduce basic observed properties. Similarly, also other authors [134] conclude that there appears to be additional physics driving Intra Cluster Medium (ICM).

In the following, we also mention some at first sight not so obviously relevant properties of astrophysical plasmas, which might well be of relevance, in particular when they are seen collectively :

a) The entropy excess [135, 136], along with the estimated short cooling time compared with the age of the medium [117], provided evidence for some kind of non-gravitational heating of the ICM. Most viable sources of heating seem to be insufficient in bringing the ICM to the observed entropy level, i.e., there is an energy crisis [137].

b) The unusually low metallicity [3), i.e. low elemental abundance [132, 136, 139, 140,

11) It is not unreasonable to assume that axions or other axion-like particles dominate the potential wells of astrophysical objects like Galaxies, or Clusters of Galaxies where dark matter was first localized by Zwicky in 1933 [128. In order to make a rough estimate of their luminosity, we use for the axion lifetime $(\tau)$ and the total mass $(\mathrm{M})$ of a group of Galaxies the values $\tau \approx 10^{17} \mathrm{sec} \approx 3$ Gyears and $\mathrm{M}=10^{13} M_{\odot} \approx 10^{67} \mathrm{erg}$, respectively. Thus, the derived luminosity is $\mathrm{L}_{x} \approx 10^{67} / 10^{17} \approx 10^{50} \mathrm{erg} / \mathrm{s}$. For comparison, the observed values are at $\mathrm{L}_{x}^{o b s} \approx 10^{42} \mathrm{erg} / \mathrm{s}$. Therefore, even if the assumed axion abundance and/or their decay rate is by several orders of magnitude smaller, X-rays from such huge astrophysical agglomerates can still provide a sensitive signature for the putative unstable particles.

12) Sersic $159-03$ with $: \mathrm{L}_{x} \approx 10^{45} \mathrm{erg} / \mathrm{s}$ below $2 \mathrm{keV}$, redshift $\mathrm{z}=0.056$, radial size of the $\mathrm{X}$-ray emitting region $\approx 800 \mathrm{kpc}$; the maximum iron abundance (=: metallicity) is $\mathrm{Z}=0.55 \times Z_{\odot}$ in the central part 132, decreasing exponentially outwards $\left(Z_{\odot}=\right.$ solar metallicity). The dominant temperature is $\mathrm{T} \approx 2.6$ $\mathrm{keV}$; a cooler component has $\mathrm{T}=(0.8 \pm 0.2) \mathrm{keV}$ and contributes by $\sim 2 \%$ in $\mathrm{X}$-rays to the total $\mathrm{X}$-ray luminosity.

The soft excess phenomenon may be a common occurence in Galaxy Clusters [133].

13) For example : a) the metallicity of the nearby Galaxy NGC 1291 measured recently with CHANDRA is $\left.\mathrm{Z}=(0.04 \pm 0.02) \times \mathrm{Z}_{\odot} 138 ; \mathrm{b}\right)$ groups of Galaxies have $\mathrm{Z}=(0.06-0.15) \times \mathrm{Z}_{\odot}$, which should be compared with $\mathrm{Z}=(0.2-0.3) \times \mathrm{Z}_{\odot}$ found in clusters 136 . One would naively expect the opposite, since the ratio of stellar mass to gas mass is higher in groups than in clusters of Galaxies 136]. 
141, 142, could also come from an overestimated mass of the gas. Note that for a thermal plasma the X-ray luminosity is a measure of the gas mass. The radiative decay of captured exotica by the same gravitational well pretends additional radiating gas, resulting in a lower metallicity [4). Even though a mixture of gas temperatures could provide an alternative explanation, according to a recent review article [136], the metallicity of the intragroup medium remains an open issue 5 .

c) The observed X-ray Luminosity-to-Gas Temperature relation in clusters of Galaxies is inconsistent with the expected $L_{x} \sim T^{2}$ relation, especially for small groups of Galaxies (e.g., it was found that $L_{x} \sim T^{8}$, or, $L_{x} \sim T^{5}$ ) [136]. Again, X-ray emission from gravitationally trapped massive axion-like particles can cause -in principle- any discrepancy [5). Note that a significant steepening appears to occur below $\sim 1 \mathrm{keV}$. The maximum deviation appears for groups of Galaxies with $\mathrm{T} \approx 0.3 \mathrm{keV}$, and this was considered as an indication for non-gravitational heating of the plasma [136, 144] [17; also, the estimated excess entropy associated with preheating corresponds to a similar temperature [146, 147]. Furthermore, it seems that the central regions of small Clusters and groups of Galaxies require a larger non-gravitational energy injection of $\sim 1 \mathrm{keV}$ per particle [148] 18). Thus, the axion scenario can accommodate the required additional energy source and the X-ray luminosity 136.

\subsection{Gravitationally trapped axions as a source of solar X-rays}

If the solar X-rays observed near the Earth originate from two-photon decay of axions produced in the Sun core, then the space around the Sun becomes a source of X-rays. In this case one expects a correlation between the solar X-ray flux, the axion density and the axion mean lifetime.

In this scenario the mean axion decay length must be much shorter than the SunEarth distance, because we know that most of the solar X-rays originate from a region near the solar surface. So, a mean axion lifetime $\tau_{a}$ of the order of one minute, or shorter, is needed. The $a \rightarrow \gamma \gamma$ decay rate is 149

$$
\tau_{a}^{-1}=\frac{g_{a \gamma \gamma}^{2} m_{a}^{3}}{64 \pi}
$$

where $g_{a \gamma \gamma}$ is the $a-\gamma-\gamma$ coupling constant and $m_{a}$ is the axion mass. For masses around

14) Note also that most elliptical and other early type Galaxies possess a hot diffuse interstellar medium $(\mathrm{T} \approx 0.5-1 \mathrm{keV})$. Equally puzzling with their low metallicity is the observed strong disagreement with the expected radiation cooling (cooling time $\leq 10^{8}$ years). In addition, there are no obvious sources of gas heating [142].

15) A group is a small cluster of galaxies.

16) The X-ray temperature from clusters of Galaxies measures also the depth of the gravitational well (given mainly by the dark matter), providing thus a crucial link between the physics of the plasma and that of the dark matter condensations [143]. If the decay or any other process associated with some exotica gives rise to the emission of X-rays, then, this link will be falsified depending on the intensity of these additional photons.

For example, for the central $\sim 200 \mathrm{kpc}$ of a Cluster of Galaxies a significant mass discrepancy between $\mathrm{X}$-ray and gravitational lensing methods seems to exist 145]: $\frac{\text { lensing mass }}{X-\text { ray mass }} \approx 2$. The mass $(M)$ enclosed within a radius $R$ is given by the relation $M(R) \sim R^{2} \cdot T$. Thus, from a modified plasma temperature $T$ the derived mass will be wrong accordingly. Interestingly, the two methods yield consistent result for large radii.

17) Probably even prior to its entry into the dark halos.

18) Within the axion scenario, this higher value should require more axions, i.e. more dark matter at the central region, implying a deeper gravitational well, which is not so unreasonable to happen. 
$1 \mathrm{keV}$ and a mean lifetime of 1 minute $g_{a \gamma \gamma}$ is $\sim 1.5 \times 10^{-3} \mathrm{GeV}^{-1}$. With such a value the mean free path for $a \rightarrow \gamma$ conversions by inverse Primakoff effect inside the Sun $(a Z \rightarrow \gamma Z$, see ref. [150]) is much shorter than the Sun radius and no axions can emerge from the Sun 151.

This inconsistency can be avoided by assuming that the main source of solar X-rays consists of accumulated long-lived axions, which are gravitationally trapped in closed orbits around the Sun. In this scenario $g_{a \gamma \gamma}$ can be small and, therefore, the axion interaction mean free path in the Sun becomes extremely long (see below in this section). Axions with lifetimes as long as the present age of the solar system $\left(T_{s} \approx 4.6 \mathrm{~Gy}\right)$, or longer, are acceptable because sooner or later they all decay in the vicinity of the Sun by definition. However, it is obvious that in this framework axions must not have a unique mass value, because in this case the trapped axions have very low velocities (lower than the escape velocity, $v_{e s c}=6.175 \times 10^{5} \mathrm{~m} / \mathrm{s}$ ), and they decay to X-rays which are almost mono-energetic. In addition, Peccei-Quinn axions from the Sun are expected to be relativistic, and, they escape from the gravitational field of a Star like our Sun.

In order to investigate this scenario we have used a simulation program based on the Kaluza-Klein (KK) axion model described in ref. [79]. In this model the lightest axion is identified with the conventional Peccei-Quinn axion and there is an infinity of heavier KK excitations separated in mass by $1 / R$, where $R$ is the compactification radius (of the order of $\left.1 \mathrm{eV}^{-1}\right)$.

In this model axions are produced by the mechanisms of photon coalescence $(\gamma \gamma \rightarrow$ a) or Primakoff effect $(\gamma Z \rightarrow a Z)$. The total solar axion luminosity $L_{a}$ (namely the rate of solar energy produced in the form of axions) is given by

$$
L_{a}=A \cdot L_{\odot} \cdot\left(\frac{g_{a \gamma \gamma}}{10^{-10} \mathrm{GeV}^{-1}}\right)^{2} \cdot\left(\frac{R}{k e V^{-1}}\right)^{\delta}
$$

where A is a numerical coefficient, $L_{\odot}=3.85 \times 10^{33} \mathrm{erg} / \mathrm{s}$ is the standard solar luminosity, and $\delta$ is the number of extra dimensions. We use $\delta=2$ and $R=10^{3} \mathrm{keV}^{-1}$ [79]. With this choice the numerical values of $\mathrm{A}$ and the average energies of the produced axions $\left(\bar{E}_{a}\right)$ are given in Table 1.

In our simulation axions with a continuous mass distribution between 0.01 and 20 $\mathrm{keV}$ are isotropically generated at different radii inside the Sun and are traced through the Sun by numerical integration of the equations of motion. The radial dependence of the solar temperature and density are taken from ref. [152]. At the Sun surface only axions with velocities $v$ below the escape velocity $v_{e s c}$ are considered further. Axions with $v>v_{\text {esc }}$ are discarded because they leave the solar system before decaying. Typical orbits of trapped axions are shown in Fig. 4.

The fractions of trapped axions for the two production mechanisms are given in Table 1 for the two production mechanisms. The trapping fraction is much smaller for axions produced by Primakoff effect, because in this case the target is at rest and it is more difficult to produce low momentum axions.

Fig. 5 shows the velocity distribution at production for axions produced by the mechanism of photon coalescence. The trapped axion mass distributions for the two mechanisms are shown in Fig. 6.

We note that in this model it is assumed that the electric charges are isolated and the initial state photons are massless. Both assumptions are not correct because the effective photon mass in the Sun core is given by the plasma energy [150], which is typically of the order of $300 \mathrm{eV}$. A non-zero photon mass is likely to affect the results of our simulation 
especially for gravitationally trapped axions which are produced with low velocities by definition. A more correct model should take this effect into account. Our intention here is to limit our study to a qualitative assessment of this scenario.

The radial density of the axions gravitationally trapped around the Sun is reconstructed from the distribution of the parameters describing the elliptical orbits outside the Sun. In this scenario the total number of trapped axions is an increasing function of time

$$
N_{a}(t)=R_{a} \tau_{a}\left(1-e^{-t / \tau_{a}}\right),
$$

where $R_{a}$ is the trapped axion production rate under the simplifying assumption of a steady Sun. Obviously, both $R_{a}$ and $\tau_{a}$ depend on the axion mass $m_{a}$ and on the coupling constan $g_{a \gamma \gamma}$. The present axion decay rate is then

$$
D_{a}\left(T_{s}\right)=R_{a}\left(1-e^{-T_{s} / \tau_{a}}\right)
$$

For a given value of $g_{a \gamma \gamma}$ we can predict the present density of trapped solar axions as a function of the distance from the Sun using Eqs. (1), (2) and (3). From this distribution, using Eq. (4) we predict the present solar X-ray spectrum on Earth and the apparent solar X-ray luminosity $\left(L_{x}\right)$ by calculating the X-ray flux from axion decay through a spherical surface of 1 AU radius centred at the Sun. We then determine the value of $g_{a \gamma \gamma}$ by requiring that our predicted X-ray luminosity be equal to the experimentally reconstructed one, i.e. $L_{x}^{2-8 k e V} \sim 10^{23} \mathrm{erg} / \mathrm{s}$ (this value corresponds to the solar X-ray spectrum reconstructed for the ASCA/SIS detector at Sun minimum and integrated over the energy interval from 2 to $8 \mathrm{keV}$ [16] (see also below)). This procedure gives $g_{a \gamma \gamma}=9.2 \cdot 10^{-14} \mathrm{GeV}^{-1}$. With such a value of $g_{a \gamma \gamma}$, the axion interaction cross section via the Primakoff effect is below $\sim 10^{-54} \mathrm{~cm}^{2}$ [150], corresponding to a mean free path much larger than the total trapped axion flight path even for the age of the Universe. The apparent X-ray luminosities and total axion luminosities obtained from this value of $g_{a \gamma \gamma}$ are given in Table 1. Fig. 7 shows the trapped axion density as a function of the distance from the Sun (for $g_{a \gamma \gamma}=9.2 \cdot 10^{-14} \mathrm{GeV}^{-1}$ ). The predicted X-ray spectrum is displayed in Fig. 8 . We note that in this scenario, and with the value of $g_{a \gamma \gamma}$ given above, the total solar axion luminosity (for both production mechanisms) is $L_{a}=6.1 \times 10^{32} \mathrm{erg} / \mathrm{s}$ (see Table 1 ), which is $\sim 16 \%$ of the standard solar luminosity $L_{\odot}$.

Table 1: List of parameter values

\begin{tabular}{|l|c|c|}
\hline & Photon coalescence & Primakoff effect \\
\hline \multicolumn{1}{|c|}{$f_{\text {trap }}$} & $9 \times 10^{-8}$ & $5 \times 10^{-11}$ \\
\hline $\mathrm{E}_{x}^{2-8 \mathrm{keV}}(\mathrm{erg} / \mathrm{s})$ & $1.0 \times 10^{23}$ & $2 \times 10^{19}$ \\
\hline$A$ & 0.067 & 0.12 \\
\hline $\bar{E}_{a}(\mathrm{erg})$ & $1.1 \times 10^{-8}$ & $6.2 \times 10^{-9}$ \\
\hline$L_{a}(\mathrm{erg} / \mathrm{s})$ & $2.18 \times 10^{32}$ & $3.91 \times 10^{32}$ \\
\hline
\end{tabular}

It is known that any exotic energy loss process in the Sun is overcompensated by an increased consumption of nuclear fuel in the core and results, therefore, in a temperature increase in the core [153]. Until recently, this temperature was mainly constrained by helioseismology [154, excluding any exotic energy loss process much in excess of the 
$0.2 L_{\odot}$ level. However, the SNO experiment has very recently measured the total neutrino flux from ${ }^{8} B$ decay in the solar core independently of the neutrino flavour [155]. This flux, $\Phi_{\nu}=(6.4 \pm 1.7) \times 10^{6} / \mathrm{cm}^{2} \mathrm{~s}$, provides a more stringent constraint to the temperature of the solar core $(T=(15.74 \pm 0.19) \mathrm{MK})$ and, therefore, to the energy loss rate from any exotic process, which is now limited to $(0.037 \pm 0.063) L_{\odot}$ [154]; i.e., the value of $L_{a}=0.16 L_{\odot}$ is still allowed within $2 \sigma$. Because of the rather crude assumptions involved in our generic model, we do not consider this limit as a serious disagreement with the scenario suggested here. One day, if axions or other particles with similar couplings are discovered and neutrino data are more accurate, they can provide very important mutual constraints.

We also note that the axion mean lifetime is much longer than the age of the solar system: as an example, for an axion mass of $5 \mathrm{keV}$ we find $\tau_{a}=1.25 \times 10^{20} \mathrm{~s}$ $\approx 4000$ Gyears. Such a lifetime is actually quite short for conventional axions. An axion related solar X-ray luminosity - as it is advocated in this work- contains then the whole Sun history since its birth (or even before?). With these values for $L_{a}, \tau_{a}$ and the mean axion velocity being $v \approx 0.6 c$ (Fig. 5), we calculate, for comparison, the X-ray luminosity within $1 \mathrm{AU}$ around the Sun coming from escaping axions decaying in flight :

$L_{x}^{2-8 k e V}($ escaping axions $) \approx \frac{10^{3} \mathrm{~s}}{10^{20} \mathrm{~s}} \times 6.1 \cdot 10^{32} \mathrm{erg} / \mathrm{s} \approx 10^{16} \mathrm{erg} / \mathrm{s}$. This makes only $10^{-7}$ of the X-ray luminosity expected from the decay of the same but trapped solar axions (see Table 1).

An additional comparison is of interest. If an axion related luminosity of $0.16 L_{\odot}$ applies to all stars, and the axion lifetime is $\sim 400 \times$ the age of the Universe, this gives a ratio

$$
\frac{\text { total photon energy from axions }}{\text { total photon energy }} \approx \frac{0.16}{400} \approx 0.4 \cdot 10^{-3}
$$

From the wide band cosmic energy density spectrum [156] the ratio between the $2-10 \mathrm{keV}$ range and the visible band is $\sim 2$ to $3 \times 10^{-3}$. Resolved $\mathrm{X}$-ray sources account for $\sim 80 \%$ of the cosmic hard X-ray background (XRB) 105. However, only $\sim 40 \%$ of the hard X-ray sources (mainly AGN) are luminous and the rest have faint or, in some cases, undetectable, optical counterparts (see Mushotzky et al. in ref.[110]). Then, for the remaining (diffuse) component this ratio becomes $\sim 10^{-3}$, which is consistent with the axion-to-photon energy ratio $\left(\sim 0.4 \cdot 10^{-3}\right)$ of this work. Interestingly, our generic axion model along with the assumption that the Sun is representative for all stars in the Universe agrees well with the cosmic energy density spectrum.

\subsection{Discussion}

In the following we compare the predictions of the axion scenario simulation with solar X-ray data, suggesting also how X-ray observatories can be utilized in this field. In so doing, we go a step further from the primary evidence based on the temperature/density distributions in the solar atmosphere, where the temperature rises outwards instead of decreasing, and it even exceeds $10^{6} \mathrm{~K}$. We refer to the quiet Sun, i.e. non-flaring Sun, during the solar minimum (this does not imply that the 'active' Sun is not of potential interest). Other effects of possible relevance are adressed too. Thus :

1) The simulated gravitational capture rates (see Table 1) for the two axion production mechanisms are rather reasonable, in particular for the $\gamma \gamma$-coalescence mode. The integrated axion luminosities $\left(L_{a}\right)$ are not inconsistent with the solar luminosity $\left(L_{\odot}\right)$ 154.

2) The shape of the reconstructed solar X-ray spectrum (see Fig. 9) is consistent with 
the predicted one from axion decays for energies above $\sim 1 \mathrm{keV}$ (Fig. 8). The bulk of the observed X-rays is, however, in the sub-keV range. From Fig. 9 the solar luminosity in the 2 to $8 \mathrm{keV}$ band is a factor of $\sim 10^{2}$ lower than that between 0.5 and $2 \mathrm{keV}$, i.e. with $L_{x}^{0.5-2 \mathrm{keV}} \sim 10^{25} \mathrm{erg} / \mathrm{s}$ at solar minimum [16], it follows that $L_{x}^{2-8 \mathrm{keV}} \sim 10^{23} \mathrm{erg} / \mathrm{s}$.

3) In the reconstructed solar X-ray spectrum (Fig. 9), the soft X-ray energy "excess" below 1 to $2 \mathrm{keV}$ might be due to a possible electron Bremsstrahlung process, which can produce axions via the axion-to-electron coupling [150]. Taking into account the results of our simulation with the Primakoff effect, it is not unreasonable to assume that KK-axions from this reaction might have a restmass distribution around the energy of the corresponding PQ-axions of $\sim 0.8 \mathrm{keV}$, i.e. $E_{\gamma}=m_{K K} / 2 \approx 0.4 \mathrm{keV}$. Their capture rate should also be comparable with the Primakoff reaction, i.e. much smaller than the $\gamma \gamma$-coalescence mode (see Table 1 ).

4) Recently, in the vicinity of the Earth, the XRB radiation in the 2 to $8 \mathrm{keV}$ region has been measured by the CHANDRA X-ray observatory [158. Its flux is 19

$$
L_{x}^{2-8 \mathrm{keV}} \approx 10^{-11} \mathrm{erg} / \mathrm{s} \cdot \mathrm{cm}^{2} \cdot \mathrm{deg}^{2} .
$$

Let us assume that this flux comes entirely from the decay of trapped axions at 1 AU from the Sun. Then, from this flux, part, or even the bulk, of the reconstructed solar X-ray luminosity at 2 to $8 \mathrm{keV}$ should be reproduced, but not (much) more, provided the X-rays from the Sun are axion-related, and their space distribution is given in Fig. 7. Then, the isotropic axion decay inside a cone with one square degree opening and $\sim 50 R_{\odot}$ height should reproduce the luminosity given by Eq. (8); the X-ray contribution from further away becomes more and more negligible, because of the decreasing axion density. With simple calculations we arrive at an upper limit of the "specific X-ray luminosity" $(S)$ in the 2 to $8 \mathrm{keV}$ range of

$$
S \approx 5 \cdot 10^{-20} \mathrm{erg} / \mathrm{s} \cdot \mathrm{cm}^{3}
$$

This rate at 1 AU from the Sun, multiplied by a factor of $\sim 10^{9}$ (following Fig. 7), gives the corresponding value near to the solar surface. The estimated effective volume around the Sun, where most of the trapped particles are expected to be (see Fig. 7), is $\sim 3 \cdot 10^{33} \mathrm{~cm}^{3}$. The integrated X-ray luminosity $\left(L_{x}^{2-8 k e V}\right)$ from the Sun should be

$$
L_{x}^{2-8 \mathrm{keV}} \approx 3 \cdot 10^{33} \mathrm{~cm}^{3} \times 5 \cdot 10^{-20} \mathrm{erg} / \mathrm{s} \cdot \mathrm{cm}^{3} \times 10^{9} \approx 1.5 \cdot 10^{23} \mathrm{erg} / \mathrm{s} .
$$

This order-of-magnitude estimate is somehow surprisingly. Because, under the assumption that the bulk of the measured diffuse 2 to $8 \mathrm{keV}$ X-rays are related to trapped solar axion decays in the vicinity of the Earth according to our simulation, we have reproduced with Eq. (7) the experimentally reconstructed solar X-ray luminosity in that energy range (see Fig. 9 and [16]). Such a "coincidence" over as much as 9 orders of magnidute can still be accidental; by comparison, the $R^{-2}$ law gives instead a change by a factor of $\sim 10^{5}$. At present, we only conclude that these two rates, differing by several orders of magnitude, are fully consistent with each other, applying the same axion scenario over $1 \mathrm{AU}$, which is a very big distance

19) The corresponding intensity of the XRB measured with ROSAT [85] is $L_{x}^{0.1-2 \mathrm{keV}} \approx 0.4 \cdot 10^{-11} \mathrm{erg} / \mathrm{s} \cdot \mathrm{cm}^{2} \cdot \mathrm{deg}^{2}$. 
for the gravitational trapping scenario of this work. A new related experimental result can change the significance of this finding accordingly. Interestingly, the same XRB measurements have also provided a clear and so far inconsistent [158, 159] field-to-field difference of $\sim 40 \%$ when pointing to the North or South celestial pole 20).

5) The generic simulation of KK-axion production of this work predicts a very specific density distribution in space (Fig. 7). This radial profile can be used for test purposes. To the best of our knowledge, such observations do not exist, at least not on purpose. Orbiting X-ray observatories with collecting areas up to $\sim 2000$ $\mathrm{cm}^{2}$, like ASCA, BeppoSAX, CHANDRA, XMM-Newton, are best suited to perform such measurements, covering a large part of the predicted radial distribution of trapped solar axions in the entire energy range $(\sim 100 \mathrm{eV}$ to $\sim 10 \mathrm{keV})$. Furthermore, an X-ray observation with the dark Moon in the telescope field-of-view should be repeated, since it allows to unambiguously extract the presumed local source of X-rays, whatever their origin might be at the end. In fact, present orbiting X-ray telescopes with very large orbits exclude possible secondary effects (e.g. scattering from the Earth atmosphere), which was definitely not the case for the ROSAT dark Moon observation.

The equivalent fiducial volume for such observations can be up to $\sim 1000 \mathrm{~m}^{3}$ (see below). This is far beyond any feasible terrestrial $4 \pi$ X-ray detector with sub$\mathrm{keV}$ threshold, indicating the potential of the orbiting X-ray observatories. Thus, without any modification, they can operate - even parasitically - as enormously sensitive detectors of radiatively decaying particles in outer space, and probably also far beyond (using for shadowing other places than the Moon). Moreover, an appropriate re-evaluation of existing runs might allow to extract relevant physics results. We suggest that such reanalyses be carried out.

6) It is worth remembering that the solar corona problem (see section 2.1) refers mainly to solar atmospheric temperatures up to $\mathrm{T} \sim 10^{6} \mathrm{~K}$, or thermal energies of, say, $3 k \mathrm{~T} \approx 0.3 \mathrm{keV}$. It is much more difficult to reconcile the emission of $\mathrm{X}$-rays from the quiet Sun with energies of $\sim 2$ to $8 \mathrm{keV}$ with conventional (solar) physics (Fig. 9). In addition, one should also notice the wide and non-thermal shape of the reconstructed solar X-ray energy distribution above $\sim 1 \mathrm{keV}$; our simulated solar axion spectra (Fig. 8) are rather similar, while reprocessing effects in the Sun atmosphere smear out the original X-ray spectrum, in particular towards lower energies (see Section $2.13)$ ).

Since the reconstructed solar X-ray spectrum, in particular below $\sim 1 \mathrm{keV}$, can not be reproduced completely, we mention some other possible effects, which can be involved:

a) Reprocessing of the absorbed higher energetic X-rays (see Section 2.13 )) can explain, for example, only $\sim 1 \%$ of the "excess" intensity at $\sim 0.5 \mathrm{keV}$ (note $L_{x}^{0.5-2 k e V} \approx 100 \times$ $\left.L_{x}^{2-8 k e V}\right)$.

b) The inner solar plasma energy is distributed around $\hbar \omega_{p l} \approx 300 \mathrm{eV}$. Even though quantitative estimates do not exist, plasma effects could have an impact on the reactions occuring inside the Sun, in particular with photon energies near $\hbar \omega_{p l}$, improving possibly the production rates and/or the capture efficiency.

20) The precise inclination of each partial pointing period relative to the connecting line X-rayObservatory-Sun will allow to find out whether this difference is possibly relevant or not for this work. 
c) Only in the vicinity of the Sun (or other similar places in the Sky) the mean spacing between the trapped exotic particles can become comparable with their de Broglie wavelength $(\lambda)$ after some accumulation time. If the bulk of these hypothetical particles is identical bosons (the KK-states are actually not), a phase transition to a Bose-Einstein Condensate (BEC) could take place. With a BEC we know that a wide range of very unusual phenomena occur [160, 161, 162, 163], like collapse, explosion, vortices, matter-wave amplification ("bosonic stimulation") 21], outward going shells, collimated jets, "miniature supernova", etc. . Such phenomena take place also in astrophysics and some of them even on our Sun. Even though these considerations are speculative, it has been argued already that axions can occur in the early Universe in the form of a Bose condensate [165], while recently it has been suggested that the dark matter in the Universe could exist in the form of BEC [166].

d) Cosmic axion Stars with strong interaction effects with magnetized media, etc., have been considered [164, 167]. This shows that high density axion clumps can interact efficiently with magnetic fields, which reach $\sim \mathrm{kGauss}$ values only near to the solar surface. In this context, we mention the observed correlation [17, 168 between solar X-ray luminosity and magnetic fields in active regions, which has been measured with the Yohkoh spacecraft.

The last two cases (c) and d)) are of potential relevance for the soft X-rays from the quiet Sun. In fact, if we apply the same reasoning as for the CHANDRA X-ray data also to the X-rays measured by the ROSAT PSPC detector, the reconstructed X-ray intensity near the solar surface is $\sim 10^{-3}$ of the reconstructed X-ray spectrum of the quiet Sun in

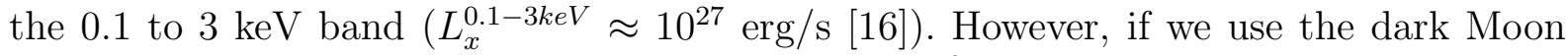
data, then the reconstructed part becomes $\sim 3 \cdot 10^{-2}$, since the considered cone height is $\approx 0.5 \times R_{\odot}$ instead of $50 \times R_{\odot}$, and, the flux is smaller by factor $\sim 3.3$ [85]. As long as the reconstructed surface X-ray luminosity is below the observed one [16], there is at least no contradiction, even though the reconstructed $\sim 3 \%$ is not an unreasonable value. However, if there is an "excess" in the reconstructed solar X-ray spectrum at low energies (Fig. 9), this could be due to local effects taking place only in the near the Sun space, e.g. cases c) and/or d) could be at work. Concerning density related effects, it is worth noticing that the mean de Broglie wavelength $(\lambda)$ of the captured low-mass axions is by a factor of $\sim 10$ larger than the one at the higher masses, since their velocity spectrum is quite similar. Since the relevant number of overlapping particles is that within a volume of $\lambda^{3}$, which increases with decreasing mass, it is reasonable to expect that density related effects might well appear pronounced at lower axion momentum and only near to the surface of the Sun. A contribution from such local effects on the Sun is obviously decoupled from that at a remote place; then, the solar surface X-ray intensity, recostructed from that at 1 AU, becomes underestimated, and this might happen with the "excess" solar soft X-rays in Fig. 9.

Of course, we consider our model as a suggestion for a possible underlying mechanism in the solar atmosphere based on solar axion production rather than a quantitative description of it. We reiterate that a detailed analysis of these and/or other processes is necessary, in order to provide a complete theoretical treatment, which is beyond the scope of this paper. Therefore, this work might be an opportunity for theory.

21) In a large density and under appropriate conditions, stimulated decays of axions to 2 photons ("axionic laser") of opposite momentum has been considered [164. 
In the following, we address a few configurations in orbit and on Earth, which seem to be the most appropriate ones to directly search for axion-like particles.

a) An orbiting X-ray telescope with the Sun being outside its FOV 22) could operate as a solar axion antenna. The dark Moon observation [85] should be repeated; during a full lunar eclipse, this might be more interesting following (X-ray) background considerations. Moreover, pointing an orbiting detector towards the dark Earth while it is in Earth's 'night' during each orbit around the Earth seems to be a very similar and attractive configuration repeating several times per day. A wide detector FOV implies a better signal-to-noise ratio, or at least a higher sensitivity to detect the radiative decay of any (solar) exotica.

We compare a wide-aperture X-ray detector orbiting at an altitude of $500 \mathrm{~km}$ and pointing towards the dark Earth with the dark Moon measurement by ROSAT covering $\mathrm{a} \sim 0.5^{\circ}$ narrow cone. The efficiency to detect the decay photons is by a factor 23) $\sim 900 \mathrm{~m}^{3} / 74 \mathrm{~m}^{3}=12$ in favour of the assumed dark Earth configuration. So far, to the best of our knowledge, the dark Earth has been observed with a narrow detector FOV 171], implying instead a suppression factor of $\sim 10^{-3}$ compared with the dark Moon observation, i.e. hopeless to have observed some signal in the past even unintentionally. Thus, the observed dark lunar emission rate of $\sim 0.15 / \mathrm{s}\left(E_{\gamma} \leq\right.$ $2 \mathrm{keV}$ ) by ROSAT 855 translates into a rate $R$ for the dark Earth configuration of

$$
R \approx 0.15 \times 12 \approx 1.8 / \mathrm{s}
$$

assuming a $200 \mathrm{~cm}^{2}$ orbiting X-ray detector at $500 \mathrm{~km}$ with $\sim 50^{\circ}$ opening angle, which implies $\mathrm{a} \sim 900 \mathrm{~m}^{3}$ effective fiducial volume within its FOV. This is actually a rather strong signal. From the dark Moon observation, the obtained modelindependent maximum axion decay rate (below $\sim 2 \mathrm{keV}$ and for non-relativistic velocities) $\mathrm{X}(\mathbf{r})$ at $1 \mathrm{AU}$ from the Sun is

$$
\mathrm{X}(|\mathbf{r}|=1 \mathrm{AU}) \approx 0.15 / \mathrm{s} \cdot 74 \mathrm{~m}^{3} \approx 2.3 \cdot 10^{-9} \text { axion decays } / \mathrm{s} \cdot \mathrm{cm}^{3} .
$$

We note that even if the solar axion scenario is not behind this particular dark Moon X-ray observation, the rate given by Eq. (9) remains valid as an upper limit for future searches for radiative decays of exotic particles in this energy range and in the vicinity of the Earth. Furthermore, following Eq. (6), the corresponding upper limit in the $\sim 2$ to $\sim 8 \mathrm{keV}$ range becomes smaller than that given by Eq. (9) by a factor of $\sim 100$.

b) An X-ray detector with $\sim 4 \pi$ acceptance operating on Earth (or, better, underground) seems to be the most adequate direct experimental approach, since it allows reconstruction of axion decays inside its fiducial volume by observing $\gamma \gamma$ coincidences. Such a detector is actually blind to any direct solar X-rays. Again,

22) X-ray telescopes avoid having the Sun in their FOV, because of the $\sim 7 \cdot 10^{9} \mathrm{X}$-rays $/ \mathrm{cm}^{2} \cdot \mathrm{s} \cdot(\sim 60-400$ $\mathrm{eV}$ ) arriving at the site of the Earth [169]. For example, ROSAT's FOV was pointing at $101^{\circ}$ away from the Sun.

23) We consider a detector surface $[170] A=200 \mathrm{~cm}^{2}$ with an opening angle of $\sim 50^{\circ}$ orbiting at $\sim 500 \mathrm{~km}$. Assuming isotropic axion decay, the effective fiducial volume covered by the detector FOV is equal to $\int \frac{2}{4 \pi r^{2}} \cdot A \cdot \cos \theta \mathrm{d} x \mathrm{~d} y \mathrm{~d} z=\frac{A}{2 \pi} \int_{0}^{2 \pi} \mathrm{d} \phi \int_{0}^{500 \mathrm{~km}} r^{2} \cdot \frac{1}{r^{2}} \mathrm{~d} r \cdot \int_{\cos 25^{\circ}}^{\cos 0^{\circ}} \cos \theta \cdot \mathrm{d}(\cos \theta)$, which is $\sim 900 \mathrm{~m}^{3}$ for a dark Earth configuration and $\sim 74 \mathrm{~m}^{3}$ for the dark Moon measurement by ROSAT [85]. $\theta$ is the angle from the normal incidence on the detector surface and the factor of 2 comes from the two photons per axion decay. 
assuming the axion scenario to explain the measured low-energy spectrum from the direction of the dark Moon [85] (Fig. 3B), and taking into account the rate derived above (see Eq. (9)), the expected coincidence rate $\mathrm{R}_{\gamma \gamma}$ should be measurable for a rather modest $\left(20 \times 20 \times 20 \mathrm{~cm}^{3}\right)$ fiducial volume:

$$
R_{\gamma \gamma} \approx 1.6 \text { coincidences } / \mathrm{d} \cdot(20 \mathrm{~cm})^{3} \approx 200 \text { coincidences } / \mathrm{d} \cdot \mathrm{m}^{3} \text {. }
$$

We are not aware of any experimental search of this type in the past. Because of the widely accepted extremely long lifetime of the 'standard' axions, such a measurement was meaningless until recently [8, 79].

Background: Uncorrelated photons are distributed uniformly over the fiducial volume while the two photons from axion decay will convert at close distance from each other. To get an order of magnitude estimate of the background rate from uncorrelated two-prong events, we take the integral single-prong event rate $\left(R_{1 \text { prong }}\right)$ as measured on the surface in a $1 \mathrm{keV}$ window at $1 \mathrm{keV}$ [172] using a Micromegas chamber [173] of dimensions $15 \times 15 \times 0.3 \mathrm{~cm}^{3}$ :

$$
R_{1 \text { prong }} \approx 1 \text { event/s }
$$

At these energies, practically all photons entering the chamber sensitive volume interact in the gas, so this rate can be used to obtain the background rate $\left(R_{b}\right)$ per $\mathrm{cm}^{3}$ inside the chamber for the same energy interval:

$$
R_{b}=R_{1 \text { prong }} /(15 \times 15 \times 0.3) \mathrm{cm}^{3} \approx 0.015 / \mathrm{s} \cdot \mathrm{cm}^{3} .
$$

If the mean photon absorption length is chosen to be $\sim 0.3 \mathrm{~cm}$, the $2 \gamma$ signal events will occur within a small cell of volume $\Delta x \Delta y \Delta z \sim 1 \mathrm{~cm}^{3}$. In a Time Projection Chamber (TPC) $\Delta x$ and $\Delta y$ are measured directly by orthogonal electrodes or pads, while $\Delta z$ can be derived from the time interval between the two signals. In a detector with a sensitive volume of $1 \mathrm{~m}^{3}$ there are $10^{6}$ cells of volume $1 \mathrm{~cm}^{3}$, hence the rate of two-prong accidental coincidences in such a detector is

$$
R_{2 \text { prong }}=10^{6} \cdot\left(R_{b}\right)^{2} \cdot \Delta t \approx 4.5 \cdot 10^{-5} \text { events } / \mathrm{s} \cdot \mathrm{m}^{3} \approx 4 \text { events } / \mathrm{d} \cdot \mathrm{m}^{3},
$$

where $\Delta t=0.2 \mu \mathrm{s}$ is the drift time over $1 \mathrm{~cm}$, assuming a standard drift velocity of $5 \mathrm{~cm} / \mu \mathrm{s}$. Of course, this rate can be reduced considerably in an underground laboratory. We note that photons of $\sim \mathrm{keV}$ energies entering the chamber will predominantly interact at small distances from the chamber walls. Thus the background rate given by Eq. (13) can be further reduced by requiring that the events occur in a fiducial volume at some distance from the walls. In addition, for non-relativistic axions, the equal energies of the two $\gamma$ 's will provide further background rejection. We note that this background varies as the third power of the photon absorption length which actually defines the cell size. It does not seem unrealistic, therefore, to reach an experimentally controllable background from two-prong events at a level well below or comparable with the expected axion signal at $\sim 1 \mathrm{keV}$.

In order to perform such a measurement, the main detector requirements are a) energy threshold as low as possible, e.g. $\sim 100 \mathrm{eV}$, in order not to miss a low-energy axion signal favoured actually by the dark Moon measurement; and b) an adequate space and energy resolution, in order to distinguish the $2 \gamma$ events from background, allowing also to implement constraints from the $a \rightarrow \gamma \gamma$ decay kinematics. Our 
preference is to photons in the sub-keV range, or even below $\sim 400 \mathrm{eV}$ (see Fig. 3 and Fig. 9 of this work, the high statistics pulse-height spectra of the soft XRB in Fig. 4 of Refs. [87, 174], the derived solar(-like) X-ray spectra (Fig. 5 in [7]), and section 4 ). However, $\mathrm{X}$-rays above $\sim 1 \mathrm{keV}$ might be not less promising. Therefore, a low-density, low-Z X-ray detector should be used. A TPC working at low pressure and/or with low-Z gas, e.g. He, appears to be a promising detector for this purpose.

\section{WHERE ELSE?}

We mention below in short some cases, where axion-like particles could be involved, and therefore, they should be followed further.

1) a) The night-time ionization in terrestrial or celestial atmospheres : the measured ionization of the Earth ionosphere at night is larger than predicted [175], requiring an extraterrestrial source of photons in the UV band. b) The ionization and heating of (nearby) interstellar medium [176, 177, 178]; the spectrum of the stellar EUV sources is too soft to explain the observed overionization of Helium with respect to Hydrogen in the Local Cloud 179, 180, which is a mystery [181.

2) Clusters of Galaxies emit in the $\sim 0.1-0.4 \mathrm{keV}$ band substantially in excess of that expected from a hot intra cluster medium at a temperature of a few keV [182]. E.g., the intense diffuse excess emission in the EUV in the Coma and Virgo Clusters of Galaxies [183, 184] is of unknown origin [5]; the spatial distribution of the EUV flux in each of these Clusters was inconsistent with that of a gravitationally bound gas [5].

3) The radiative decay of the escaping exotica outside the Sun or other places in the Sky resembles the scattering of (solar) X-rays off electrons or dust particles in near space (see Fig. 5.1 in Ref. [58]). The same reasoning can apply to similar configurations in remote interstellar space [185] and to extended X-ray sources [186]. However, the recent observations of an X-ray halo with the Chandra X-ray telescope provided unexpected results, since they do not fit the conventional dust scattering model [187. The axion scenario of this work might be the only alternative explanation.

4) Underground experiments with threshold in the sub-keV range could provide a direct detection of the putative particles. Taken into account the ROSAT efficiency and narrow energy bandwidth, the estimated rate from Eq. (9) is $\sim 0.1$ event $/ \mathrm{kg} /$ day $/ \mathrm{keV}$ below $\sim 1 \mathrm{keV}$.

5) Seyfert Galaxies $(N L S 1)$ show a giant soft X-ray excess below $\sim 1 \mathrm{keV}$ [130, 188, 189], with an X-ray luminosity of $\sim 10^{44} \mathrm{ergs} / \mathrm{s} \approx 10^{17} \times \mathrm{L}_{\odot}^{x}$ and strong variability on time scales of $\sim 1$ day [190]. A direct observation of the source size is not yet available, but a simple blackbody emission is ruled out on the basis of the inferred remarkably small size of the emission region, exceeding the Eddington limit [190].

6) The heating of the intergalactic medium, as its origin is not yet clear [191]. Also, the warm/hot intergalactic medium between Clusters of Galaxies, which has not been detected so far neither in emission nor in absorption, it makes probably a significant fraction of the baryons in the local Universe $(\mathrm{z} \leq 1-2)$ [111].

7) The large-scale diffuse X-ray emission $(\mathrm{T} \approx 6 \mathrm{keV})$ from the centre of our own Galaxy is of as yet unknown origin 192, with the giant molecular cloud Sgr B2 being the strongest diffuse X-ray source in this region [193 24). Also, the universal X-ray

24) If a diffused plasma distribution around the Galactic Centre is indeed the source of the emitted X-rays with a flux $\approx 1.1 \cdot 10^{-10} \mathrm{ergs} / \mathrm{s} \cdot \mathrm{cm}^{2} \cdot \mathrm{deg}^{2}$, then its temperature and energy density are both too 
emission of AGNs along with the observed evolution with cosmic time is of potential interest [102, 194, 195, 196, 197, 198]; the exact mechanism which produces the Xrays is not known 199. Afterall, massive black holes have the strongest gravitational effects and make $\sim 0.6 \%$ of the bulge mass of a Galaxy 197.

8) Solar "micro-events" [20], which seem to be present at all times and also in coronal holes; these are short emission enhancements (= flares) of $\sim 10^{25 \pm 2}$ ergs in quiet regions, or, localized brightenings and explosive events. Their msec variability challenges the understanding of the coronal plasma [29], but also the down-flows along with the redshifted lines, which are seen in the transition region [200, 201].

9) Solar/celestial jets [202], including the puzzling X-ray tails behind Pulsars (or even Galaxies?) 203.

\section{Conclusion}

A missing explanation of an astrophysical observation is actually suggestive to search for an exotic approach. The framework of the celebrated dark-matter physics world is a source of possible exotic solutions. In the cases considered here, a same axion-like scenario consistently explains the usually different alternative conventional solutions (if they exist at all). This scenario is not supposed to abandon globally previous models, which describe actually related findings; it can be rather complementary, providing a so far missing physics input.

A temperature (component) of a few $10^{6} \mathrm{~K}(\sim 0.3 \mathrm{keV})$, which appears in so diverse astrophysical places, such as from the solar corona to Clusters of Galaxies and probably beyond, is associated with several unexpected significant observations. In order to explain this in a combined way, we reach the conclusion that some new particles - we use massive axions as a generic example- must be involved in processes occurring inside and outside Stars. For example, relatively short-lived massive axions have been considered in theories with extra dimensions [8, 79]; the two-photon decay mode remains dominant providing theoretical support to our purely observationally/astrophysically motivated claim of celestial axion-like signatures in the $\sim \mathrm{keV}$ range. In our favoured scenario, axion-like particles escape from their place of birth, e.g., from the interior of the Sun (or that of other Stars in the Sky), get gravitationally trapped and decay in outer space.

Alternatively, a more or less isotropic radiative decay of other hypothetical particles, e.g. massive neutrinos, could in principle also explain the astrophysical observations considered here. Only laboratory experiments could clarify this issue. We give a (theoretically) unbiased parameter space how and where to directly search for such exotica. Fortunately, the two-photon decay mode allows to have a very high detection sensitivity inside a large TPC, because of the much suppressed uncorrelated two-prong background events within a small distance, narrow time and same energy. High-performance low-threshold detectors developed primarily for high-energy physics experiments can also be utilized for this kind of astro-particle physics.

Following the reasoning for the suggested axion-like scenario, we should also notice that the previous failed searches [204, 205] for axions converted to X-rays inside the external solar/stellar magnetic fields do not contradict this work. At first, this missing signal can be due to an accordingly small coupling strength and/or the required very small axion restmass. Independent on this actually unknown value, the "conventional" Primakoff

high for the plasma to be gravitationally bound around the Galactic Centre 193. In fact, the point sources account for only $\sim 10 \%$ of the total flux in the $2-10 \mathrm{keV}$ energy range, indicating strong diffuse X-ray emission (see Ebisawa et al., in ref. 193). 
effect should result to radially outwards emitted X-rays, excluding a self-irradiation of the Sun, which we consider as the cornerstone of the reasoning of this work. The tentative solar Kaluza-Klein model provides reasonable gravitational capture rates, but it fails to completely explain the low energy part of the reconstructed solar X-ray spectrum. However, other possible sources like the solar Bremsstrahlung-axions seem to have reasonably low energy, but with a smaller gravitational capture rate. For astrophysical standards, the encountered discrepancies are actually not particularly large.

Finally, the estimated axion density due to gravitational trapping by the Sun can exceed a critical value, which is necessary for the appearance of a BEC, with unforeseen implications. The continuous dynamic coronal phenomen might be a manifestation of such processes. Thus, the predicted particles orbiting around the Sun can become an invaluable clue to physics beyond the standard (solar) model, explaining first of all the as yet mysterious properties of the chromosphere and the corona. The gravitational trapping of massive particles emitted by the Star itself provides a mechanism for the appearance of boson clumps around a Star.

In conclusion, the strongest and rather direct evidence in favour of the axion scenario comes from the sofar unexplained solar corona related observations like its heating mechanism, its narrow interface to the chromosphere, the chromosphere itself, together with the striking similarities of the temperature/density profiles with the Sun-irradiated Earth atmosphere. The recently experimentally reconstructed solar X-ray spectra combined with X-ray measurements from orbiting observatories are the first potential direct signatures for this work. They show how these orbiting instruments can directly search for decaying particles in (near) outer space, providing also the expected maximum rate in future investigations of this kind. The relevant new parameter introduced by this work is the as yet disregarded elongation angle of the X-ray Telescope relative to the Sun. The other astrophysical observations we have addressed, in particular when they are seen combined, provide an additional piece of evidence, probably of not minor importance at the end.

\section{Acknowledgements}

Constructive criticisms by Alexander Dolgov have stimulated us to go deeper into the axion scenario. We wish to thank Apostolos Pilaftsis for stimulating discussions, and Jim Rich for his comments and suggestions that we have taken into account in this paper. We also acknowledge the help by Thomas Papaevangelou. We thank Joachim Trümper for the permission to use Figures 2 and 3 from Ref. [85]. We are grateful to Judith Lean for indicating to us the references about the Earth ionosphere and for allowing us to use Fig. 1 from Ref. [33]. We also wish to thank Giovanni Peres for allowing us to use Figure 9 of this work and Salvatore Orlando for providing us with the original numerical values. We also thank Tullio Basaglia, Gilda Leoni, Marie-Jeanne Servettaz and Jens Vigen from the CERN library for the great help provided in finding many not so easily accessible articles, and some of them even twice. 


\section{References}

[1] S. Bowyer et al., ApJ. 526 (1999) 10, and references therein.

[2] A.L. Melott, Sov. Astron. 28 (1984) 478; J. Ipser, P. Sikivie, Phys. Rev. Lett. 50 (1983) 925; M.S. Turner, F. Wilczek, A. Zee, Phys. Lett. 125 (1983) 35; S. Bowyer, A.R.A.A. 29 (1991) 59.

[3] A. De Rújula, S.L. Glashow, Phys. Rev. Lett. 45 (1980) 942; D.W. Sciama, Phys. Rev. Lett. 65 (1990) 2839, ApJ. 364 (1990) 549, ibid. 409 (1993) L25; ibid. 488 (1997) 234.

[4] K. Abazajian, G.M. Fuller, W.H. Tucker, ApJ. 562 (2001) 593.

[5] S. Bowyer, J.J. Drake, S. Vennes, A.R.A.A. 38 (2000) 231.

[6] M.S. Turner, Phys. Rep. 197 (1990) 67.

[7] M. Güdel, E.F. Guinan, S.L. Skinner, ApJ. 483 (1997) 947.

[8] K.R. Dienes, E. Dudas, T. Gherghetta, Phys. Rev. D62 (2000) 105023.

[9] O. Bertolami, Astroph. Space Sci. 261 (1999) 225; A. Loeb, Phys. Rev. D48 (1993) 3419; Z. Berezhiani, A. Drago, Phys. Lett. B473 (2000) 281.

[10] R.C. Henry et al., ApJ. 516 (1999) L49.

[11] V.K. Narayanan et al., ApJ. 543 (2000) L103; see also P. Bode, J.P. Ostriker, N. Turok, ApJ. 556 (2001) 93.

[12] J.H.M.M. Schmitt, Astron. Astroph. 318 (1998) 215.

[13] T.R. Burnight, Phys. Rev. 76 (1949) 165; H. Friedman et al., ibid. 83 (1951) 1025; R. Tousey, K. Watanabe, J.D. Purcell, ibid. p. 792.

[14] O. Podladchikova et al., Astron. Astroph. 382 (2002) 713, preprint astro-ph/0108333 (21. 8. 2001).

[15] C.E. Parnell, Astroph. Space Sci. 261 (1999) 81.

[16] S. Orlando, G. Peres, F. Reale, ApJ. 528 (2000) 524 and ibid. 560 (2001) 499; G. Peres et al., ApJ. 528 (2000) 537; F. Reale, G. Peres, S. Orlando, ApJ. 557 (2001) 906.

[17] A.A. Pevtsov, L.W. Acton, ApJ. 554 (2001) 416.

[18] K. Nandra, I.E. Papadakis, ApJ. 554 (2001) 710.

[19] P. Dmitruk, L.J. Milano, W.H. Matthaeus, ApJ. 548 (2001) 482.

[20] A.O. Benz, S. Krucker, ApJ. (20. 3. 2002).

[21] A. Fluda et al., J. Geophys. Res. 104 (A5) (1999) 9709.

[22] A.O. Benz, S. Krucker, Astron. Astroph. 341 (1999) 286.

[23] U. Feldman et al., ApJ. 518 (1999) 500.

[24] G. Peres et al., ApJ. 563 (2001) 1045.

[25] J.H.M.M. Schmitt, Cool Stars, Stellar Systems and the Sun, eds. R.A. Donahue, J.A. Bookbinder, ASP Conf. Series 154 (1998) 463.

[26] B. Haisch, J.H.M.M. Schmitt, P.A.S.P 108 (1996) 113.

[27] J.B. Birker, Solar Physics 148 (1993) 43.

[28] M.J. Aschwanden et al., ApJ. 535 (2000) 1027.

[29] M.J. Aschwanden, A.I. Poland, D.M. Rabin, A.R.A.A. 39 (2001) 175.

[30] G.A. Doschek, J.T. Mariska, ApJ. 560 (2001) 420.

[31] P.G. Judge, T.D. Tarbell, K. Wilhelm, ApJ. 554 (2001) 424.

[32] R. Wolfson et al., ApJ. 539 (2000) 995.

[33] J. Lean, A.R.A.A. 35 (1997) 33.

[34] D. Alexander, J. Geophys. Res. 104 (A5) (1999) 9701.

[35] R. Wolfson et al., ApJ. 529 (2000) 570 and ref.'s therein. 
[36] H.O. Evje, E. Leer, Astron. Astroph. 329 (1998) 735.

[37] H.P. Warren, A.R. Winebarger, ApJ. 535 (2000) L63 and ref,'s therein.

[38] By Earth standards the corona is truly vacuous: its density is $\sim 10^{-12} \mathrm{~g} / \mathrm{cm}^{3}$ at a height of $\sim 2000 \mathrm{~km}$ (the transition region from the chromosphere to the corona) and decreases to $5 \cdot 10^{-19} \mathrm{~g} / \mathrm{cm}^{3}$ at a distance of 3 solar radii. See the contributions by R.L. Moore, M.E. Machado, J. Zirker, E.H. Avrett, R.F. Stein, N.R. Sheeley, G.L. Withbroe in The Astronomy and Astrophysics Encyclopedia, St. P. Maran (Edit.), Van Nostrand Reinhold, New York (1992) pp. 637-642 and 850-860.

[39] K.J.H. Phillips, Plasma Phys. Control. Fusion 42 (2000) 113.

[40] T.R. Ayres, IAU Symposium 185, Kyoto, Japan, 18-22/8/1997, F.-L.Deubner et al., (eds.), Kluwer Academic Publ., Dordrecht (1998) 403.

[41] The morphology of the transition region remains unsolved [37, 201]. The question is not whether such a thin interface exist, but whether it contributes substatially to the bright emission seen in transition region lines on the solar disk (see also ref.[30]).

[42] C.U. Keller, Experientia 51 (1995) 710.

[43] A.N. Cox, Allen's Astrophysical Quantities, The Athlone Press, London (2000).

[44] J.M. Fontenla, E.H. Avrett, R. Loeser, ApJ. 406 (1993) 319.

[45] E. Marsch, C.-Y. Tu, K. Wilhelm, Astron. Astroph. 359 (2000) 381.

[46] D.E. Groom et al., The Particle Data Group, Eur. Phys. J. C15 (2000) 169; http://physics.nist.gov/PhysRefData/ $\left(E_{\gamma} \geq 1 \mathrm{keV}\right)$; http://physics.nist.gov/PhysRefData/FFast/html/form.html $\left(E_{\gamma} \geq 10 \mathrm{eV}\right)$.

[47] M.J. Aschwanden, ApJ. 560 (2001) 1035.

[48] A. Unsöld, Physik der Sternatmosphären, Springer, Berlin (1955) 666.

[49] G.M. Keating, N.C. Hsu, Geophys. Res. Lett. 22 (1993) 2751.

[50] A.F. Nagy, in Ref. 38] p. 516.

[51] G.L. Withbroe, R.W. Noyes, A.R.A.A. 15 (1977) 363.

[52] H. Hara et al., PASJ 46 (1994) 493.

[53] J. Madej, A. Różańska, Astron. Astroph. 356 (2000) 654.

[54] D.R. Ballantyne, R.R. Ross, M.N.R.A.S. (2002), preprint astro-ph/0201420 (25. 1. 2002).

[55] J. Li et al., ApJ. 538 (2000) 415.

[56] G.A. Doschek et al., ApJ. 482 (1997) L109.

[57] K. Wilhelm, Adv. Space Res. 25 (2000) 1723.

[58] K.J.H. Phillips, Guide to the Sun, Cambridge University Press (1992).

[59] B.N. Dwivendi, A. Mohan, K. Wilhelm, Adv. Space Res. 25 (2000) 1751.

[60] Y.-M. Wang, Cool Stars, Stellar Systems and the Sun, eds. R.A. Donahue, J.A. Bookbinder, ASP Conf. Series 154 (1998) 131.

[61] S.W. Kahler, J.C. Raymond, J.M. Laming, 9th Intern. Solar Wind Conf., ed. S.R. Habbal et al., AIP Conf. Proc. 471, Nantucket/Massachusetts (1999) 685.

[62] K.P. Dere, R.A. Howard, G.E. Brueckner, Adv. Space Res. 25 (2000) 1837.

[63] K.P. Raju et al., ApJ. 543 (2000) 1044.

[64] D.J. Helfand, E.C. Moran, ApJ. 554 (2001) 27.

[65] C.L. Olson and U. Schumacher, Springer Tracks in Modern Physics, Collective Ion Acceleration 84 (1979) 1.

[66] J. Bosser, ed., Proc. Beam Cooling and Related Topics, CERN 94-03 (1994).

[67] M. Güdel, ApJ. 480 (1997) L121.

[68] V. Kashyap, J.J. Drake, ApJ. 503 (1998) 450.

[69] B. Haisch, J. Schmitt, SKY \& TELESCOPE 98 (Oct. 1999) 46. 
[70] E.R. Priest et al., ApJ. 539 (2000) 1002.

[71] C.J. Schrijver, M.J. Aschwanden, ApJ. 566 (2002) 1147.

[72] A.S. Eddington, M.N.R.A.S. 86 (1926) 320.

[73] E.A. Milne, M.N.R.A.S. 87 (1926) 43.

[74] T.A. Fleming, ApJ. 504 (1998) 461.

[75] J. Edelestein, R.S. Foster, S. Bowyer, ApJ. 454 (1995) 442.

[76] W. Becker, J. Trümper, Astron. Astroph. 341 (1999) 803.

[77] W. Becker, Adv. Space Res. 25 (2000) 647.

[78] T.A. Fleming, K. Werner, M.A. Barstow, ApJ. 416 (1993) L79; K. Werner et al., Astron. Astroph. 307 (1996) 860; K. Werner, MPE Report 263 (1996) 205.

[79] L. Di Lella, A. Pilaftsis, G. Raffelt, K. Zioutas, Phys. Rev. D62 (2000) 125011.

[80] S.D. Kawaler, M. Dahlstrom, Amer. Scient. 88 (2000) 498.

[81] P. Bergeron, ApJ. 558 (2001) 369.

[82] D. Schönberner, T. Driebe, T. Blöcker, Astron. Astroph. 356 (2000) 929 and ref.'s therein.

[83] J.J. Drake et al., ApJ. (20. 6. 2002) in press, preprint astro-ph/0204159 (9. 4. 2002). See also NASA News Release : 02-082.

[84] J. Trümper, Adv. Space Res. 2 (4) (1983) 241.

[85] J.H.M.M. Schmitt, S.L. Snowden, B. Aschenbach, G. Hasinger, E. Pferrermann, P. Predehl, J. Trümper, Nature 349 (1991) 583.

[86] B.C. Flynn et al., Geophys. Res. Lett. 25 (1998) 3253.

[87] S.L. Snowden et al., ApJ. 454 (1995) 643.

[88] M.J. Freyberg, see IAU Colloquium in Ref. 94] p. 113.

[89] a) The extragalactic diffuse X-ray background was one of the first discoveries in Xray astronomy by R. Giacconi, H. Gursky, F.P. Paolini, B.B. Rossi, Phys. Rev. Lett. 9 (1962) 439. b) The existence of a galactic emission below $1 \mathrm{keV}$ was first observed by C.S. Bowyer, G.B. Field and J.E. Mack, Nature 217 (1968) 32. c) The existence of an interstellar galactic medium of $\sim 10^{6} \mathrm{~K}$ was first predicted by L. Spitzer, ApJ. 124 (1956) 20.

[90] J. Trümper, Q. J. R. astr. Soc. 33 (1992) 165.

[91] C. Day, Physics Today 53 (5) (2000) 18; W. Cui et al., ApJ. 468 (1996) 117.

[92] I.M. McHardy et al., M.N.R.A.S. 295 (1998) 641; E. Boldt, D. Leiter, Nucl. Phys. B S 38 (1995) 440; E.C. Moran et al., ApJ. 556 (2001) L75; D.M. Alexander et al., AJ 122 (2001) 2156, preprint astro-ph/0107450 (23. 7. 2001).

[93] Y. Suto et al., ApJ. 461 (1996) L33.

[94] S. Park, K. Ebisawa, ApJ. 547 (2001) 777. For more ref.'s see Proc. of the IAU Colloquium No. 166, The Local Bubble and Beyond, D. Breitschwerdt, M.J. Freyberg, J. Trümper (Eds), Garching 21-25/4/1997, Berlin, Springer (1998).

[95] B. Welsh, F. Crifo, R. Lallement Astron. Astroph. 333 (1998) 101.

[96] R.J. Egger, M.J. Freyberg, G.E. Morfill, Space Sci. Rev. 75 (1996) 511;

D. Breitschwerdt et al., ibid. 78 (1996) 183; K.D. Kuntz, S.L. Snowden, F. Verter, ApJ. 484 (1997) 245; S.L. Snowden et al., ApJSS 128 (2000) 171.

[97] D.M. Sfeir et al., Astron. Astroph. 346 (1999) 785, and ref.'s therein;

J. Má́z-Apellániz, ApJ. 560 (2001) L83.

[98] Q. Wang et al., ApJ. 374 (1991) 475.

[99] E.g. C.S. Bowyer et al., in Ref. [89], J. Kerp, Astron. Astroph. 289 (1994) 597.

[100] K.D. Kuntz, S.L. Snowden, R.F. Mushotzky, ApJ. 548 (2001) L119; 
[101] C.R. Barber, T.P. Roberts, R.S. Warwick, M.N.R.A.S. 282 (1996) 157; R.S. Warwick, T.P. Roberts, Astron. Nachrichten 319 (1998) 59; Q.D. Wang, T. Ye, New Astronomy 1 (1996) 245; L.-W. Chen, A.C. Fabian, K.C. Gendreau, M.N.R.A.S. 285 (1997) 449; R. Gilli, M. Salvati, G. Hasinger, Astron. Astroph. 366 (2001) 407. [102] T. Miyaji, G. Hasinger, M. Schmidt, Adv. Space Res. 25 (2000) 827.

[103] T. Miyaji et al., Astron. Astroph. 334 (1998) L13.

[104] A.N. Parmar et al., Astron. Astroph. 345 (1999) 611; A. Vecchi et al., Astron. Astroph. 349 (1999) L73.

[105] A. Kushino et al., PASJ. (2002) in press, preprint astro-ph/0204224v3 (12. 5. 2002); see also W.B. Brandt et al., preprint astro-ph/0202311 (16. 2. 2002).

[106] M. Güdel, CERN Courier 40 (7) (2000) 17.

[107] G.M. Voit, A.E. Evrard, G.L. Bryan, ApJ. 548 (2001) L123 and ref.'s therein.

[108] S. Park et al., ApJ. 476 (1997) L77; S.L. Snowden et al., ApJ. 430 (1994) 601; Y. Sato, H. Tsunemi, K. Yamashita, Astroph. Space Sci. 250 (1997) 53.

[109] G. Hasinger et al., Astron. Astroph. 275 (1993) 1, Astron. Nachr. 318 (1997) 329.

[110] G. Hasinger et al., Astron. Astroph. 329 (1998) 482; R.F. Mushotzky et al., Nature 404 (2000) 459; R. Giacconi et al., ApJ. 551 (2001) 624; I. Georgantopoulos et al., M.N.R.A.S. 291 (1997) 203.

[111] L.A. Phillips, J.P. Ostriker, R. Cen, ApJ. 554 (2001) L9.

[112] S.L. Snowden, M.J. Freyberg, ApJ. 404 (1993) 403.

[113] V.V. Izmodenov, R. Lallement, Y.G. Malama, Astron. Astroph. 342 (1999) L13; P. Jelinsky, J.V. Vallerga, J. Edelstein, ApJ. 442 (1995) 653; S.M. Kahn, Nature 351 (1991) 607.

[114] S.L. Snowden, in the Proc. IAU of Ref. [94] p. 103.

[115] S.L. Snowden et al., ApJ. 493 (1998) 715, ibid. 354 (1990) 211.

[116] T.J. Sumner et al., in Ref. 94 p. 333.

[117] R. Lieu et al., ApJ. 458 (1996) L5.

[118] D.A. Buote, ApJ. 548 (2001) 652.

[119] A.C. Fabian, Science 271 (1996) 1244.

[120] H. Böhringer et al., Nature 368 (1994) 828.

[121] S. Borgani, XXI Moriond Astrophysics Meeting, Galaxy Clusters and the High Redshift Universe Observed in X-Rays, Eds. D. Neumann et al. (2001), preprint astro-ph/0106471 (26. 6. 2001).

[122] C. Vignali et al., Astron. Astroph. 370 (2001) 900.

[123] J. Mather et al., ApJ. 420 (1994) 439; E.L. Wright et al., ibid. p. 450.

[124] R.C. Henry et al., A.R.A.A. 29 (1991) 89.

[125] A.C. Fabian, X. Barcoons A.R.A.A. 30 (1992) 429.

[126] B.D. Blout et al., ApJ. 546 (2001) 825.

[127] M.A. Bershady, M.T. Ressell, M.S. Turner, Phys. Rev. Lett. 66 (1991) 1398.

[128] F. Zwicky, Helv. Phys. Acta 6 (1933) 110.

[129] For example : M. Gliozzi et al., Astron. Astroph. 377 (2001) 44; T.J. Turner et al., ApJ. 561 (2001) 131; F. Frontera et al., ApJ. 561 (2001) 1006; G. Matt et al., Astron. Astroph. 377 (2002) L31, preprint astro-ph/0108508 (31. 8. 2001).

[130] P. Romano et al., ApJ. 564 (2002) 162, and ref.'s therein for previous observations with ROSAT (1997), BeppoSAX (1998) and ASCA (1998).

[131] M. Bonamente, R. Lieu, J.P.D. Mittaz, ApJ. 561 (2001) L63; see also F. Durret et al., Astron. Astrophys. (2002) in press, preprint astro-ph/0204345 v2 (21. 5. 2002).

[132] J.S. Kaastra et al., Astron. Astroph. 365 (2001) L99. 
[133] M. Bonamente et al., preprint astro-ph/0205473 (27. 5. 2002).

[134] J.J. Bialek, A.E. Ervard, J.J. Mohr, ApJ. 555 (2001) 597.

[135] M. Loewenstein, ApJ. 532 (2000) 17; A. Mahdavi et al., ApJ. 534 (2000) 114.

[136] J.S. Mulchaey, A.R.A.A. 38 (2000) 289, and ref.'s therein.

[137] P. Tozzi, Proc. APS Conf. Series (Sesto 2001), preprint astro-ph/0109072 (5. 9. 2001); see also F. Governato, ASP Conf. Series 2002, Sesto Pusteria, preprint astroph/0201493 (29.1.2002).

[138] J.A. Irwin, C.L. Sarazin, J.N. Bregman, ApJ. 570 (1. 5. 2002).

[139] P. Tozzi, Proc. APS Conf. Series (Vulcano 2001), preprint astro-ph/0107150 (9. 7. 2001).

[140] X.-Y. Xia et al., ApJ. 564 (2002) 196.

[141] A.M. Read, T.J. Ponman, D.K. Strickland, M.N.R.A.S. 286 (1997) 626.

[142] H. Xu et al., preprint astro-ph/0110013 (3. 10. 2001);

see also F. Brighenti, W.G. Mathews, preprint astro-ph/0203409 (22. 3. 2002).

[143] A. Cavaliere, N. Menci, P. Tozzi, ApJ. 484 (1997) L21.

[144] F. Brighenti, W. G. Mathews, ApJ. 553 (2001) 103.

[145] S.-J. Xue, X.-P. Wu, preprint astro-ph/0204510 (30. 4. 2002), and ref.'s therein.

[146] T.J. Ponman, D.B. Cannon, J.F. Navarro, Nature 397 (1999) 135.

[147] E.J. Lloyd-Davies, T.J. Ponman, D.B. Cannon, M.N.R.A.S. 315 (2000) 689.

[148] S. Borgani et al., ApJ. 559 (2001) L71, preprint astro-ph/0205471 (27. 5. 2002);

K.K.S. Wu, A.C. Fabian, P.E.J. Nulsen, M.N.R.A.S. 324 (2001) 95.

[149] G.G. Raffelt, Phys. Rep. 198 (1990) 1.

[150] G.G. Raffelt, Phys. Rev. D33 (1986) 897.

[151] A.D. Dolgov, private communication.

[152] J.N. Bahcall, Neutrino Astrophysics, Cambridge Unversity Press, Cambridge (1990).

[153] G.G. Raffelt, Phys. Rep. 333-334 (2000) 593.

[154] H. Schlattl, A. Weiss, G. Raffelt, Astroparticle Phys. 10 (1999) 353.

[155] Q.R. Ahmad et al., Direct Evidence for Neutrino Flavor Transformation from Neutral-Current Interactions in the Sudbury Neutrino Observatory, preprint nuclex/0204008 v2 (9. 5. 2002).

[156] M.G. Hauser, E. Dwek, A.R.A.A. 39 (2001) 249; G. Hasinger, preprint astroph/0001360 (20. 1. 2000).

[157] J.J. Drake et al., ApJ. 545 (2000) 1074.

[158] L.L. Cowie et al., ApJ. 566 (2002) L5, and ref's therein.

[159] G. Hasinger et al., Astron. Astroph. 365 (2001) L45.

[160] F. Dalfovo et al., Rev. Mod. Phys. 71 (1999) 462.

[161] M.H. Anderson et al., Science 269 (1995) 198.

[162] W.P. Reinhardt, S.B. McKinney, Physica Scripta T90 (2001) 202.

[163] G.P. Collins, Scient. Amer. 283 (December 2000) 68.

[164] I.I. Tkachev, Phys. Lett. B191 (1987) 41; C.J. Hogan, M.J. Rees, Phys. Lett. B205 (1988) 228; E.W. Kolb, I.I. Tkachev, Phys. Rev. Lett. 71 (1993) 3051; T.W. Kephart, T.J. Weiler, Phys. Rev. D52 (1995) 3226 and Nucl. Phys. B (Proc. Suppl.) 72 (1999) 54.

[165] E.J. Daw, Nucl. Phys. B (Proc. Suppl.) 72 (1999) 124; M. Srednicki, The Eur. Phys. J. C15 (2000) 143.

[166] W. Hu, R. Barkana, A. Gruzinov, Phys. Rev. Lett. 85 (2000) 1158.

[167] E.g., A. Iwazaki, Progr. Theor. Phys. 101 (1999) 1253, Phys. Lett. B486 (2000) 147, Phys. Rev. D60 (1999) 025001. 
[168] G.H. Fisher et al., ApJ. 508 (1998) 885.

[169] V. Krasnopolsky, ICARUS 128 (1997) 368.

[170] A.N. Parmar et al., Astron. Astroph. Suppl. 136 (1999) 407.

[171] K. Makishima et al., PASJ 48 (1996) 171.

[172] Measurement performed in Saclay in collaboration with the group of Y. Giomataris, using a Micromegas chamber [173] previously exposed to a beam. Charged particles were rejected.

[173] Y. Giomataris, Ph. Rebourgeard, J.P. Robert and G. Charpak, Nucl. Instr. Meth. in Phys. Res. A376 (1996) 29.

[174] E. Pfeffermann, U.G. Briel, IEEE Trans. NS-39 (1992) 976.

[175] D.F. Strobel et al., J. Geophys. Res. 79 (1974) 3171; Planet. Space Sci. 28 (1980) 1027.

[176] J.D. Slavin, P.C. Frisch, ApJ. 565 (2002) 364.

[177] R.J. Reynolds, L.M. Haffner, S.L. Tufte, ApJ. 525 (1999) L21.

[178] S.M. Viegas, RevMexAA (Serie de Conferencias) (2000), preprint astro-ph/0102392 (22. 2. 2001) and ref.'s therein.

[179] J. Vallerga, ApJ. 497 (1998) 921.

[180] R.J. Raud ApJ. 501 (1998) 137.

[181] E.B. Jenkins et al., ApJ. 538 (2000) L81.

[182] M. Bonamente, R. Lieu, J.P.D. Mittaz, ApJ. 547 (2001) L7.

[183] T.W. Berghöfer, S. Bowyer, E. Korpela, ApJ. 535 (2000) 615.

[184] A.C. Fabian, Science 275 (1997) 48 and ref.'s therein; K. Mitsuda et al., ApJ. 353 (1990) 480; P. Predehl et al., Science 257 (1992) 935.

[185] D.P. Rolf, Nature 302 (1983) 46; J.S. Mathis, C.-W. Lee, ApJ. 376 (1991) 490.

[186] A.M. Soltan et al., Astron. Astroph. 349 (1999) 354.

[187] R.K. Smith, R.J. Edgar, R.A. Shafer, preprint astro-ph/0204267 (16. 4. 2002).

[188] Th. Boller, New Astronomy Rev. 44 (2000) 387.

[189] K.A. Pounds, J.N. Reeves, Proc. Symposium "New Visions of the X-ray Universe in the XMM-Newton and Chandra Era", 26-30/11/2001, preprint astro-ph/0201436 (26. 1. 2002).

[190] T.J. Turner et al., ApJ. 548 (2001) L13;

G.C. Dewangan et al., preprint astro-ph/0205114 (8. 5. 2002).

[191] A.V. Kravtsov, G. Yepes, M.N.R.A.S. 318 (2000) 277 astro-ph/0004333, 24. 4. 2000); K.K.S. Wu, A.C. Fabian, P.E.J. Nulsen, ibid. p. 889 (astro-ph/9907112 v2, 11. 7. 2000).

[192] R.S. Warwick, preprint astro-ph/0203333 (20. 3. 2002).

[193] K. Koyama, Proc. ASP Conf. Series, preprint astro-ph/0108109 (7. 8. 2001) and ref's therein; H. Murakami, K. Koyama, Y. Maeda, ApJ. 558 (2001) 687; A. Senda, H. Murakami, K. Koyama, ApJ. 565 (2002) 1017; L. Sidoli et al., Astron. Astroph. 372 (2001) 651; K. Ebisawa et al., Science 293 (2001) 1633, preprint astro-ph/0203070 (6. 3. 2002); Q.D. Wang, E.V. Gotthelf, C.C. Lang, Nature 415 (2002) 148, and ref's therein.

[194] A.E. Hornschemeier et al., ApJ. 541 (2000) 49.

[195] J.-U. Fischer et al., Astron. Nachr. 319 (1998) 347.

[196] G. Hasinger et al., Astron. Astroph. 365 (2001) L45.

[197] G. Hasinger et al., in Highlights in X-ray Astronomy in Honor of Joachim Trümper's 65th Birthday, MPE Report, MPE, Garching, preprint astro-ph/9901103 v2 (14. 1. 1999). 
[198] F. La Franca et al., ApJ. 570 (1. 5. 2002).

[199] R.F. Mushotzky, preprint astro-ph/0203310 (19. 3. 2002).

[200] E. Landi et al., Astron. Astroph. 357 (2000) 743.

[201] O. Wikstol, P.G. Judge, V. Hansteen, ApJ. 501 (1998) 895.

[202] E.g. : A.S. Wilson, A.J. Young, P.L. Shopbell, ApJ. 547 (2001) 740.

[203] V.M. Kaspi et al., ApJ. 562 (2001) L163; B.M. Gaensler, M.J. Pivovaroff, G.P. Garmire, ApJ. 556 (2001) L107; D. Lai, D.F. Chernoff, J.M. Cordes, ApJ. 549 (2001) 1111; D.J. Helfand, E.V. Gotthelf, J.P. Halpern, ApJ. 556 (2001) 380; G. Gavazzi et al., ApJ. 563 (2001) L23; A. Neronov et al., preprint astro-ph/0201410 (24. 1. 2002).

[204] G.G. Raffelt, Phys. Rep. 333-334 (2000) 593, and ref.'s therein.

[205] E.D. Carlson, L.-S. Tseng, Phys. Lett. B365 (1996) 193;

E.D. Carlson, Phys. Lett. B344 (1995) 245. 


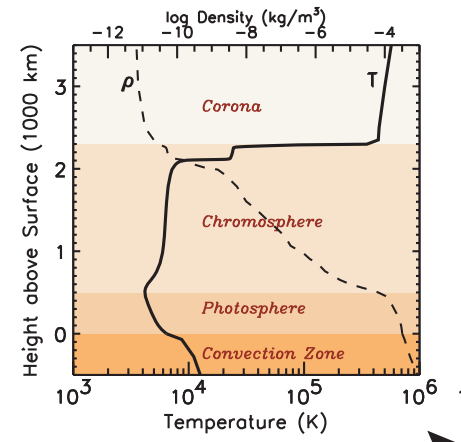

a)

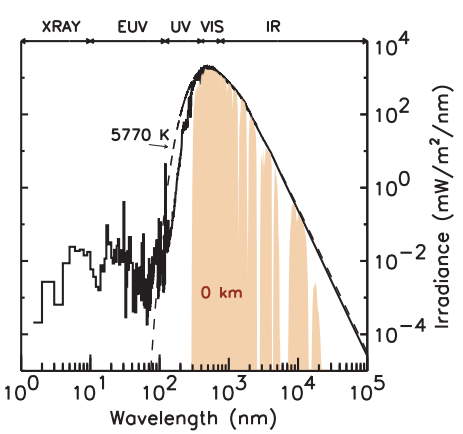

b)

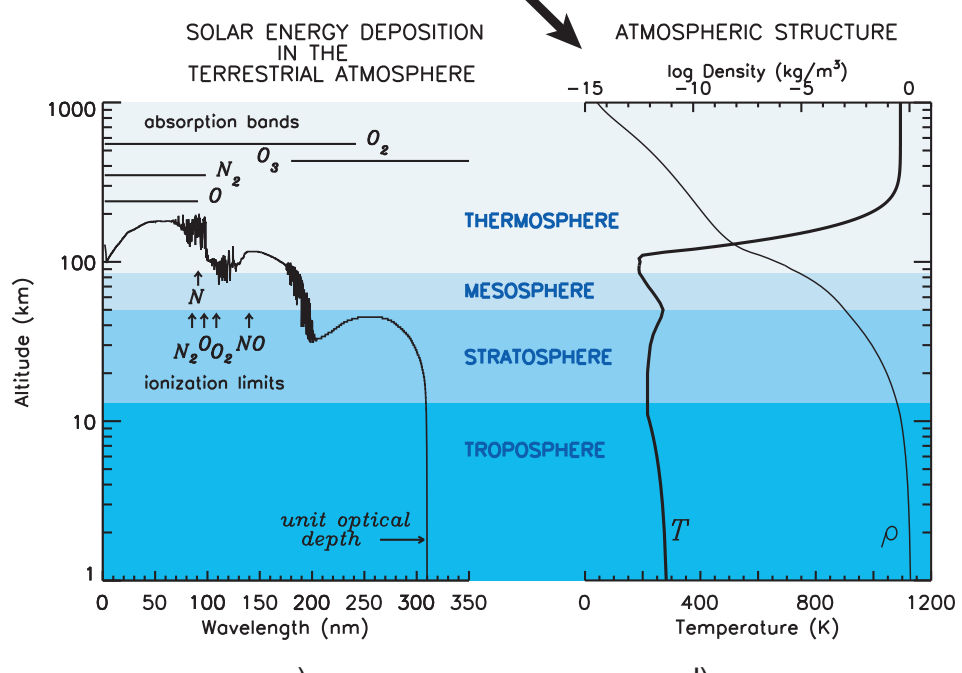

c)

Figure 1: (a) The mean temperature $(T)$ and density $(\rho)$ profiles for the solar atmosphere; (b) solar irradiance spectrum (the dashed line is the Planck shape for a temperature $T=5770 \mathrm{~K}$ ); (c) the altitude at which the Earth atmosphere attenuates the incident solar radiation by a factor $1 / e ;(\mathbf{d})$ temperature $(T)$ and density $(\rho)$ as functions of height in the Earth atmosphere. (These figures are taken from Ref. [33].) 


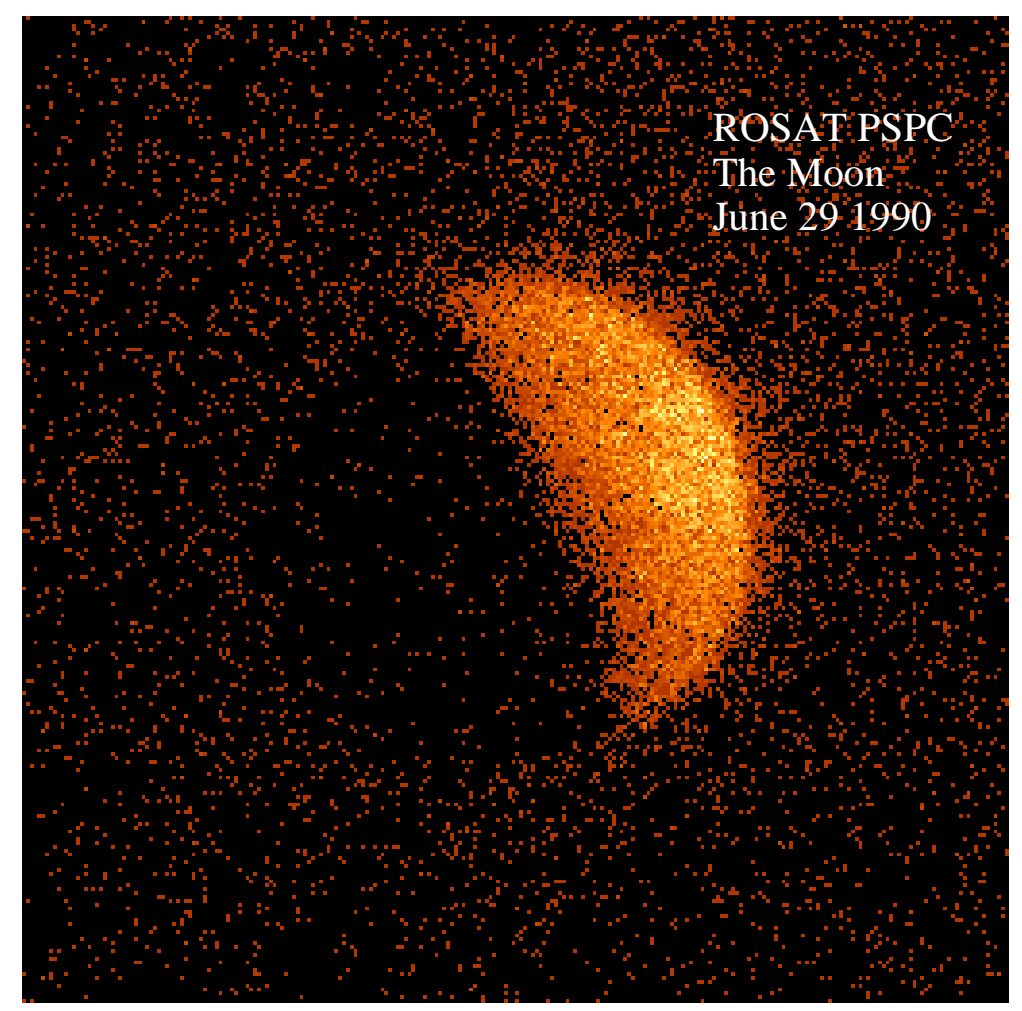

Figure 2: The X-ray photon image of the Moon as measured by ROSAT. The sunlit portion of the Moon is visible, as well as an X-ray shadow in the diffuse XRB radiation cast by the dark side of the Moon. Grey pixels denote one or two events, except in the brightest part of the crescent, corresponding to three or more counts. 


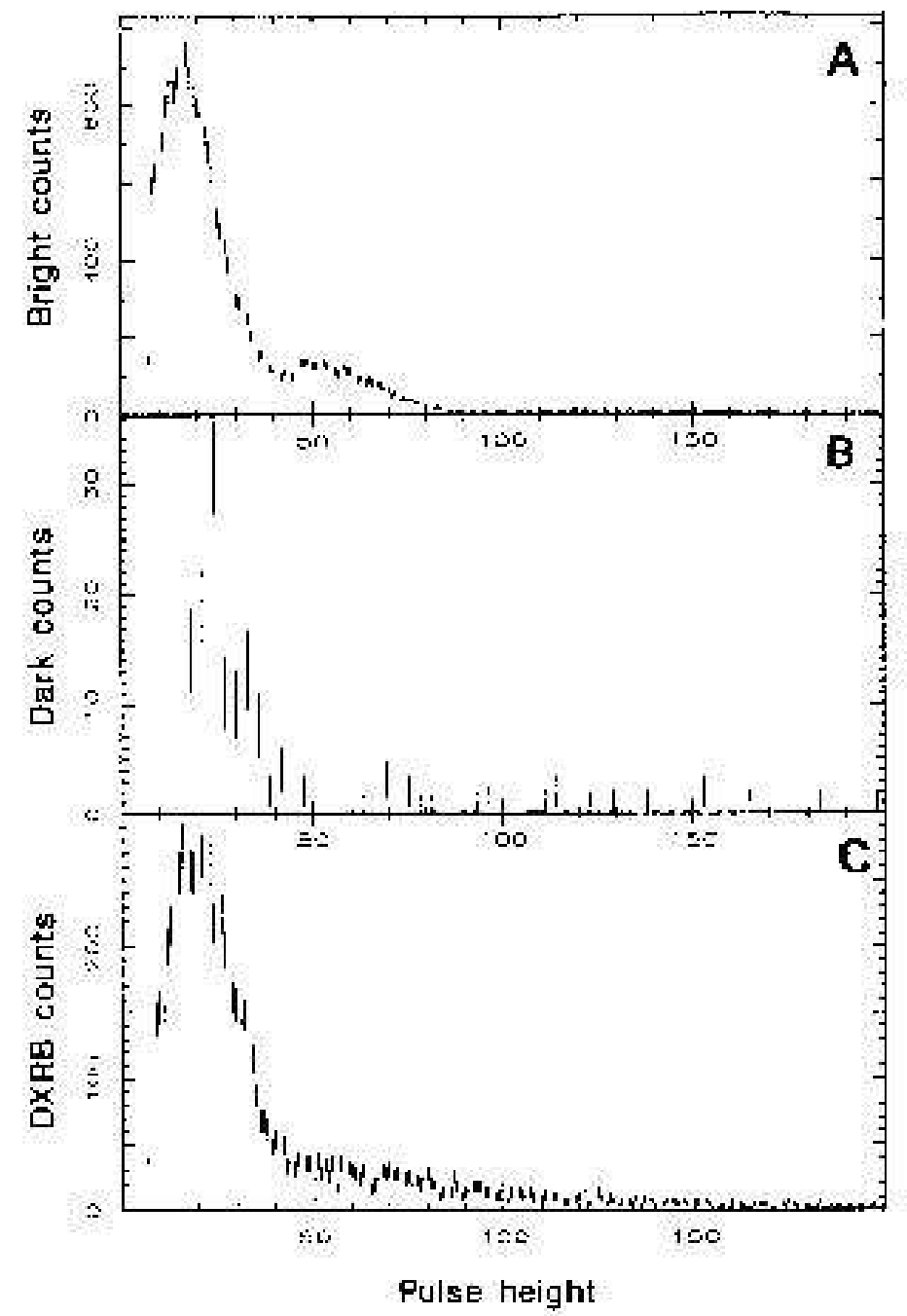

Figure 3: Measured raw pulse-height spectra from the observation in Fig. 2 (channel number multiplied by 10 gives the energy in $\mathrm{eV}$, and, the effective area of the detector system peaks at the same value of $\sim 200 \mathrm{~cm}^{2}$ around $250 \mathrm{eV}$ and $\left.1 \mathrm{keV}\right)$ : (A) X-rays from the sunlit side of the Moon; (B) X-rays from the dark side of the Moon; (C) cosmic X-ray background radiation. (Figures 2 and 3 are taken from Ref. 85). 

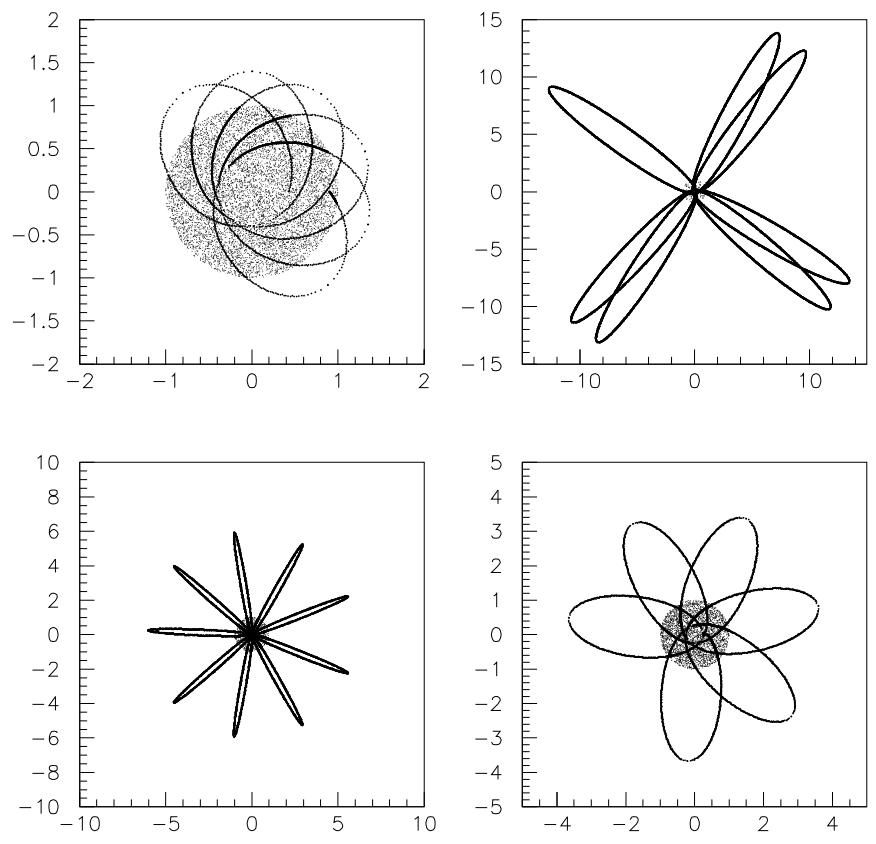

Figure 4: Typical orbits of solar axions gravitationally trapped around the Sun. The two orthogonal coordinates are given in solar radii. The shadowed region in the center of each figure outlines the solar disk. Only the first few revolutions are traced. 

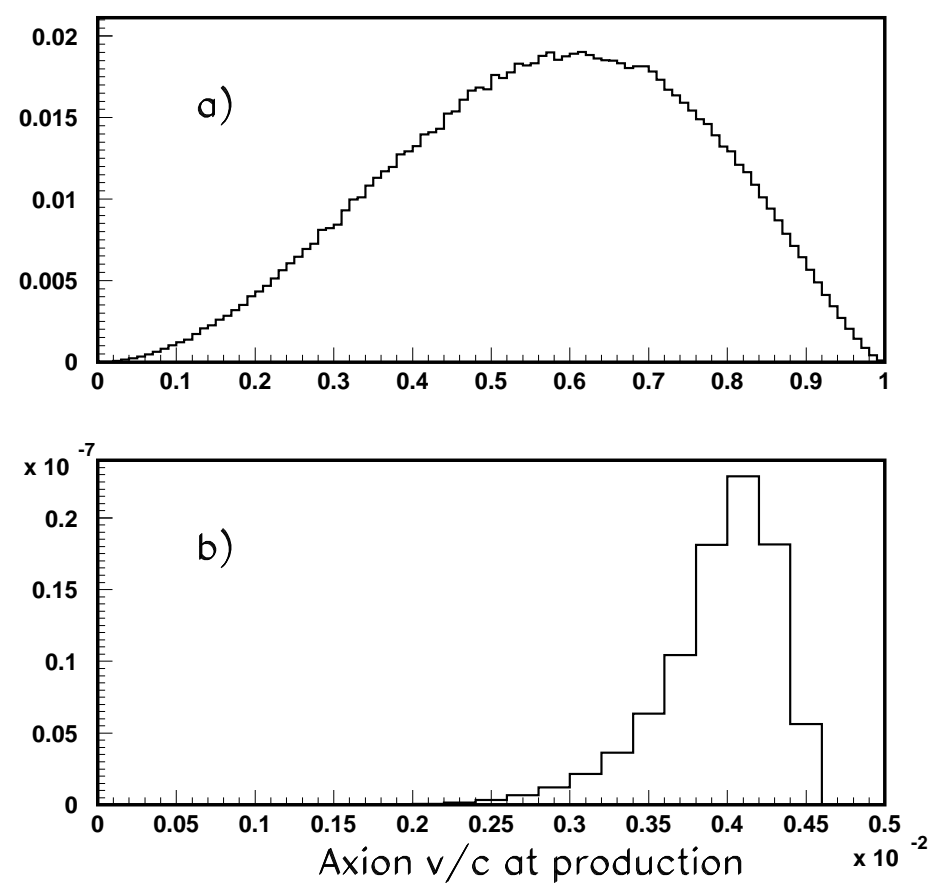

Figure 5: Velocity distribution at production for solar axions from photon coalescence : a) all axions (distribution normalised to unity); b) gravitationally trapped axions (distribution normalised to $\left.f_{\text {trap }}\right)$. 


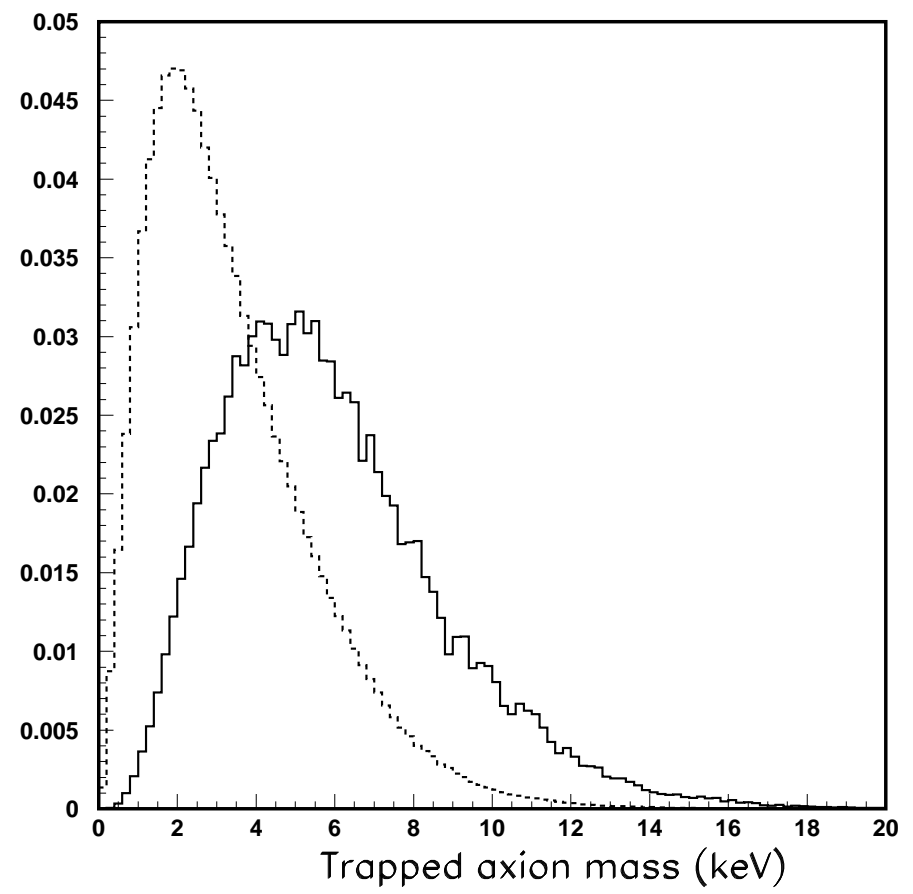

Figure 6: Mass distribution of gravitationally trapped solar axions produced by photon coalescence (full line) and by Primakoff effect (dotted line). The two curves are normalized to unit area.

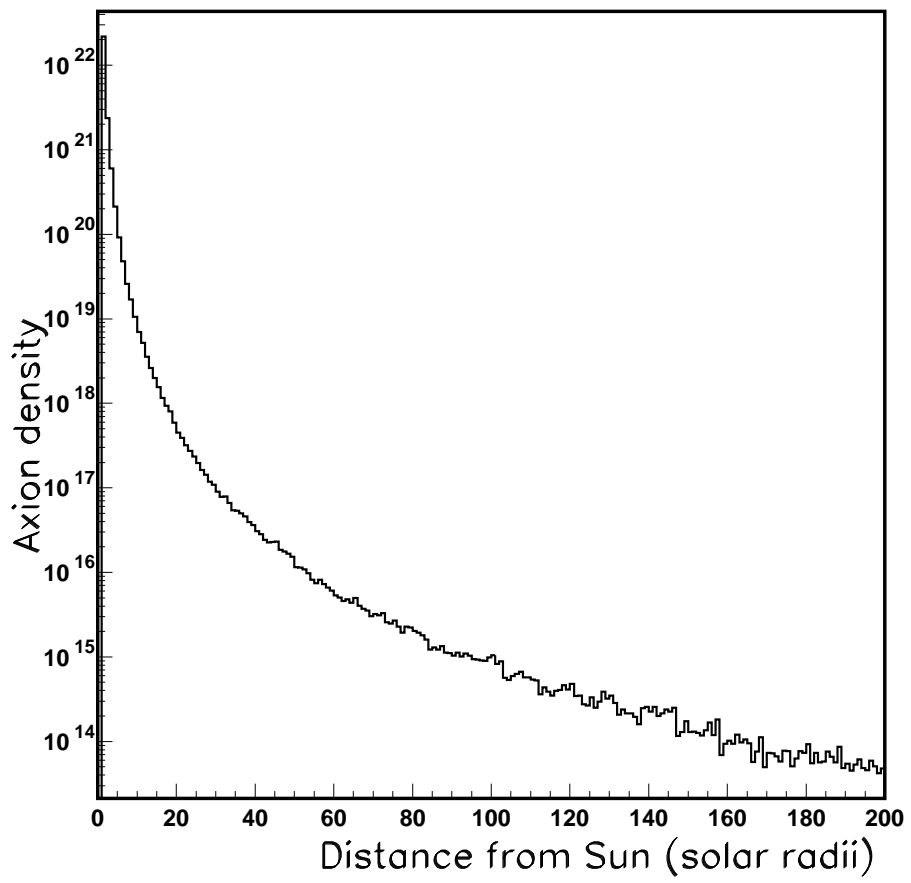

Figure 7: Present density (axions per $\mathrm{m}^{3}$ ) of gravitationally trapped axions in the region around the Sun, as a function of the distance from the Sun centre. 


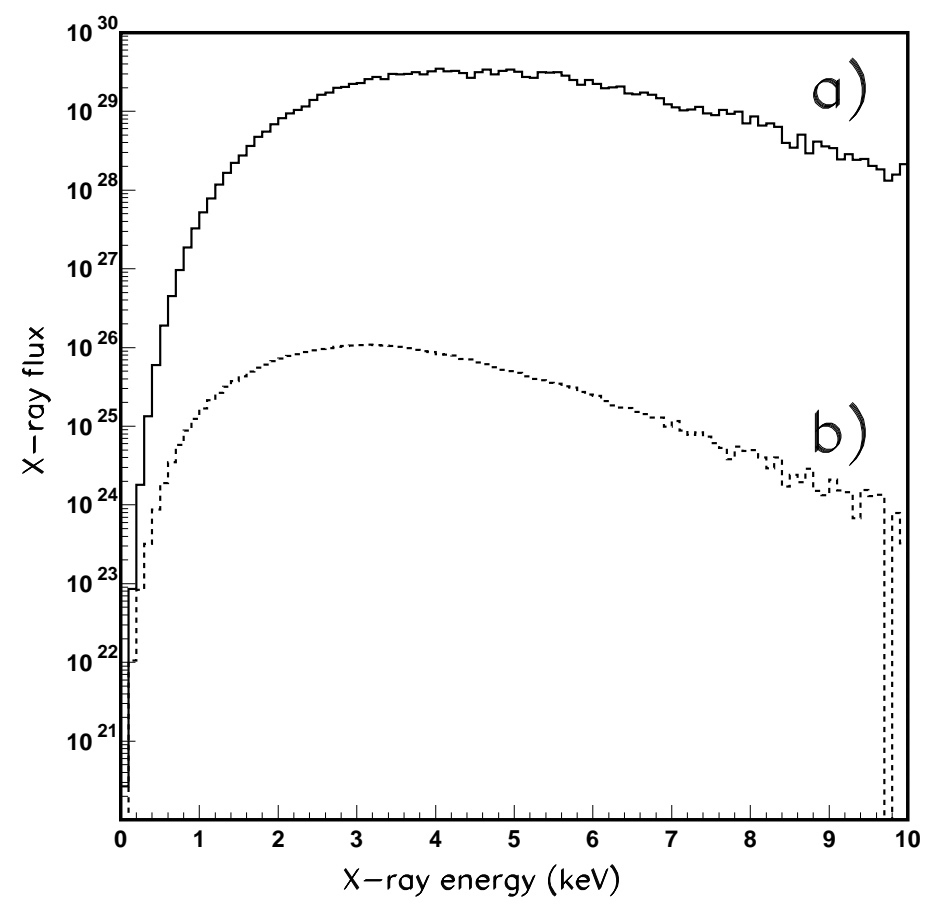

Figure 8: Expected X-ray energy spectrum from the decay of gravitationally trapped solar axions produced by a) photon coalescence and b) Primakoff effect. The two curves are normalized to the predictions of our simulations for the present age of the solar system.

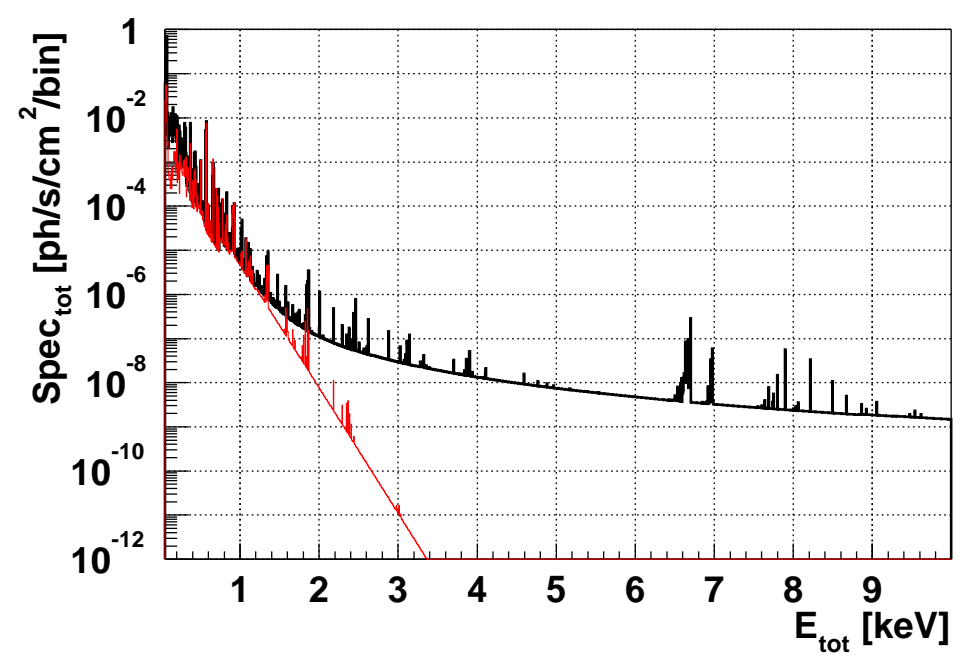

Figure 9: The solar X-ray spectrum reconstructed from the emission measure distribution $(\operatorname{EM}(\mathrm{T}))$ for the non-flaring Sun at the solar minimum [16]. A thermal component of $\sim 1.8 \mathrm{MK}$ is also shown (thin line in red). Bin size $=6.1 \mathrm{eV}$.

$(\mathrm{EM}(\mathrm{T})$ is approximately the product of the square of the electron density with the emitting volume as a function of temperature, i.e. $\operatorname{EM}(\mathrm{T})=\rho_{e}^{2}(T) \cdot V(T)$ 157). 